\title{
On the method of reflections
}

\author{
Philippe Laurent, Guillaume Legendre’’ Julien Salomon
}

June 23, 2021

\begin{abstract}
This paper aims at reviewing and analysing the method of reflections, which is an iterative procedure designed for solving linear boundary value problems set in multiply connected domains. Being based on a decomposition of the domain boundary, this method is particularly well-suited to numerical solvers relying on boundary integral representation. For both the sequential and parallel forms of the method appearing in the literature, we interpret the procedure in terms of projection operators. Using a Hilbert space setting and orthogonality, we prove the unconditional convergence of the sequential form and propose a modification of the parallel one that makes it unconditionally converging. Several examples of boundary value problems that enter such a framework are given, an alternative proof of convergence is provided in a case which does not. A few numerical tests conclude the study.
\end{abstract}

\section{Contents}

1 Introduction 2

2 Two examples of applications from the literature 3

2.1 An electrostatic analogue of the mobility problem: the sequential form . . . . . . . . . . . . . 3

2.2 An electrostatic analogue of the resistance problem: the parallel form . . . . . . . . . . . . . . . . 4

3 General formulations 5

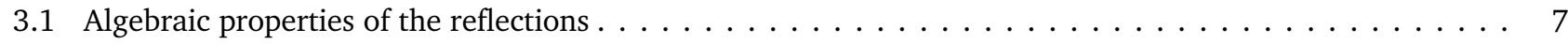

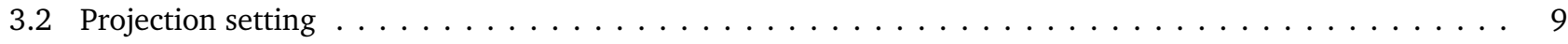

4 Convergence analysis in a Hilbert space setting 11

4.1 The orthogonal case: theoretical results $\ldots \ldots \ldots \ldots \ldots$

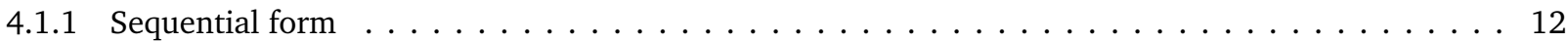

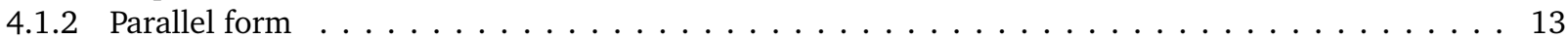

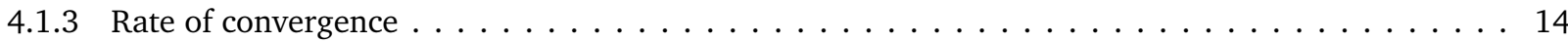

4.2 The orthogonal case: practical examples $\ldots \ldots \ldots \ldots \ldots$

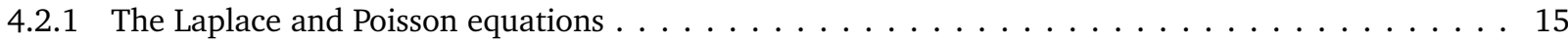

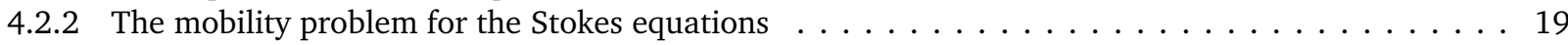

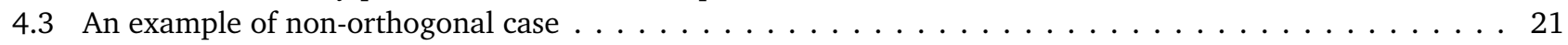

5 Numerical experiments 25

5.1 Rate of convergence in a bounded domain . . . . . . . . . . . . . . . . . . . . . . . . . . . . .

5.2 Mixed boundary conditions: a case of divergence of the sequential form . . . . . . . . . . . . 26

5.3 Influence of the distance in an unbounded domain $\ldots \ldots \ldots \ldots$

Institut de Recherche en Communications et Cybernétique de Nantes, CNRS, UMR 6597, École des Mines de Nantes, 4, rue Alfred Kastler, 44307 Nantes, France (philippe.laurent@mines-nantes.fr).

†CEREMADE, UMR CNRS 7534, Université Paris-Dauphine, Université PSL, Place du Maréchal De Lattre De Tassigny, 75775 Paris cedex 16, France (guillaume.legendre@dauphine.fr).

INRIA Paris, ANGE project-team, 2 rue Simone Iff, 75589 Paris cedex 12, France. Sorbonne Universités, Laboratoire Jacques-Louis Lions (julien . salomon@inria.fr). 


\section{Introduction}

In 1911, inspired by the method of image charges in electromagnetism, Smoluchowski [Smo11] introduced an iterative process to compute the hydrodynamic forces exerted on an assemblage of an arbitrary number of spheres falling in an unbounded viscous fluid. Later dubbed the method of reflections, this technique has subsequently been featured prominently in reference textbooks on hydrodynamics at low Reynolds number, like those by Happel and Brenner [HB83], Kim and Karrila [KK91] or Dhont [Dho96], and employed in several articles dealing with the motion of particles immersed in a viscous fluid (see for instance [Kyn59, Jon78, CK88, IB01, Wil13]). It may be described as a systematic scheme by which a (generally exterior) linear boundary value problem associated with several "objects" (e.g., particles in the case of a suspension) may be solved by computing and summing solutions of boundary value problems involving only a single object, which are called reflections. Physically speaking, one may visualise its very principle by "supposing an initial disturbance to be reflected from the boundaries involved and to produce succeedingly smaller effect with each successive reflection" [HB83, chapter 8], hence its given name.

In a more formal manner, the method exploits the superposition principle, inherent to any linear system, to follow a divide-and-conquer strategy, assuming that each of the single-object problems is somewhat easier to solve than the many-object one. In this sense, it bears some similarities to Schwarz-type domain decomposition methods (see [Gan08] for instance), which attempt to solve a (generally interior) boundary value problem by splitting it into several boundary value problems set on different, at most partially overlapping, subdomains and iterating to coordinate the solution between adjacent subdomains. There is, however, an important conceptual difference between these two approaches. Indeed, any single object problem to be solved in the method of reflections is defined on a (possibly unbounded) domain that is the interior of the complement of the considered object and thus contains the whole domain in which the main problem is set. This technique must therefore rather be seen as a boundary decomposition method.

The method of reflections was historically devised with human computers in mind, as well as tractable and explicit formulas for the reflections, which implied that the objects were few and identical, with a shape such that available analytical forms of the Stokes and Faxén laws allowed the practical approximation of the velocity field by a truncated series. With time, these restrictions, coupled with the fact that a large number of terms may be required in the truncated expansion to obtain an accurate approximation (most notably when the objects are close to each other), made its use less attractive. Nevertheless, since the method in itself does not rely on the manner in which the boundary value problems are solved, it became clear, with the advent of electronic computers, that methods using a numerical discretization (more advantageously those based on boundary integral representation) could be employed, and even combined ${ }^{1}$ with the aforementioned analytical methods, to efficiently address problems involving numerous objects of arbitrary shape, size or type of imposed boundary condition.

Despite a number of papers concerned with applications (see [IB01, ML11, BGJ ${ }^{+} 12$, Wil13] for instance), it appears that the method of reflections has seldom been studied from a mathematical perspective. One must cite the pioneering work of Luke [Luk89], in which the convergence of the original form of the method applied to the solution of the socalled mobility problem in a bounded domain is investigated theoretically. By formulating the problem in an appropriate Hilbert space setting and interpreting the method in terms of orthogonal projection operators, Luke proved that the method always converges to the solution of the problem and that its convergence rate is linear if a geometric property between the subspaces associated with the projection operators holds. More recently, Traytak [Tra06] studied a variant of the method applied to the solution of a Dirichlet problem in a three-dimensional unbounded domain complementary to a set of spheres. Resorting to analytical techniques, he established necessary and sufficient conditions of convergence with respect to the radii of the spheres and the distances between their respective centres. These two important contributions notwithstanding, it appears that a fully developed mathematical theory of the method of reflections is still lacking.

Furthermore, we may add that a few missed facts have led to some misunderstandings on the part of the scientific communities in which the method is used. For instance, when Ichiki and Brady [IB01] state, citing the work of Luke, that "while the convergence of the method of reflections has been proven for the mobility problem, the convergence for the resistance problem is an open question." and subsequently give a "counter-example" to the convergence of the method for configurations involving more than two objects, they somehow undermine their discovery by failing to notice that the method of reflections considered in their work differs from the one studied by Luke. It was also empirically observed by Wilson [Wil13] that the convergence behaviour of the method may depend significantly on the type of boundary value problem considered, and Luke stated that his convergence proof does not extend to other types of boundary conditions or in the case of an unbounded domain, leaving open a number of questions. In the present work, we show that convergence can indeed be established for several kinds of boundary value problems using the same mathematical tools. By doing so, we aim at broadening the original analysis of Luke and propose a unified theoretical framework for

\footnotetext{
${ }^{1}$ One can for instance check the reference $\left[\mathrm{BGJ}^{+} 12\right]$, in which different solution methods are used depending on the nature of the considered one-object problem.
} 
the analysis of the existing forms of the method of reflections.

The outline of the paper is the following. Using a well-known analogy between Stokesian hydrodynamics and electrostatics, we first review in Section 2 the different instances of the method of reflections found in the literature on a couple of toy problems involving the Laplace operator. This exposition leads to a more formal presentation of two versions of the method, applied to the solution of an abstract boundary value problem. The first form, called sequential in the present work, appears to be the original one from an historical perspective. The second one, which we call parallel, is, to the best of our knowledge, a variant independently introduced by Golusin in 1934 [Gol34] and seems to be the form of the method featured the most prominently in the literature. In Section 3, the method is recast in a loose functional framework, which allows to derive some algebraic properties of the iterative process and to interpret it in terms of projection operators. Section 4 is devoted to the convergence analysis of the method in a Hilbert space setting. Unconditional convergence results are obtained, for both the sequential form and a modification of the parallel form of the method, whenever the considered boundary value problem can be solved by the method of orthogonal projection [Wey40, Vis49], and several examples of applications entering such a context are given. Finally, some numerical experiments are presented in Section 5.

\section{Two examples of applications from the literature}

In this section, we describe two forms of the method of reflections commonly found in the literature. The method is indeed typically considered in the context of hydrodynamics at low Reynolds number to solve two distinct types of problems for flows involving hydrodynamic interactions among particles, respectively called the mobility problem and the resistance problem (see [KK91] for instance). Both are boundary value problems based on the Stokes equations for an incompressible flow of a Newtonian fluid. In the former, the forces and torques are to be determined for specified particle velocities in the ambient fluid, while in the latter the particles' forces and torques are prescribed in the ambient fluid and the velocities are unknown. However, to simplify the presentation, we exploit an existing analogy between Stokesian hydrodynamics and electrostatics, recalled by Luke [Luk89], in which the Stokes equations are replaced by the Laplace equation, and the fluid velocity by the electrostatic potential.

\subsection{An electrostatic analogue of the mobility problem: the sequential form}

The method of reflections, as introduced by Smoluchowski [Smo11], was studied by Luke [Luk89] when used to solve the mobility problem in hydrodynamics. The electrostatic analogue of this problem consists in determining the electric potential in a container $\Omega$ in $\mathbb{R}^{d}$ ( $d$ being an integer strictly greater than 2 ), such that its value is constant, but unknown, on the surfaces of $N$ conducting objects $O_{j} \subset \Omega, j$ in $\{1, \ldots, N\}$, and the corresponding amount of electric charge on each of these surfaces is known, that is: given $N$ real numbers $Q_{j}, j$ in $\{1, \ldots, N\}$, find the scalar function $u$ and the $N$ real numbers $c_{j}, j$ in $\{1, \ldots, N\}$, which verify

$$
\begin{aligned}
& -\Delta u=0 \text { in } \Omega \backslash \cup_{j=1}^{N} \overline{O_{j}}, \\
& u=c_{j} \text { on } \partial O_{j}, j=1, \ldots, N, \\
& \int_{\partial O_{j}} \frac{\partial u}{\partial \boldsymbol{n}}(x) \mathrm{d} S(x)=Q_{j}, j=1, \ldots, N,
\end{aligned}
$$

where $\Delta$ denote the Laplace operator, $\frac{\partial u}{\partial \boldsymbol{n}}$ is the trace of the normal derivative of $u$ on the considered boundary, $\boldsymbol{n}$ being the outward-pointing unit normal vector to the boundary, and $\mathrm{d} S$ stands for the volume form on each of the considered hypersurfaces.

Such a non-local boundary condition is sometimes said to be of the fourth type ${ }^{2}$ [Sho77, Chapter III] or called an equivalued surface boundary condition or a total flux boundary condition [Li89]. It arises in various applications (see the examples given in [Li89]).

Note that the domain $\Omega$ may or may not be bounded. In the latter case, one should prescribe an additional condition at infinity for the problem to be well-posed. In what follows, we assume that the container is bounded and that a homogeneous Dirichlet condition is imposed on its boundary:

$$
u=0 \text { on } \partial \Omega \text {. }
$$

In order to solve the resulting boundary value problem, the method of reflections generates a sequence $\left(u^{(k)}\right)_{k \in \mathbb{N}}$ of approximations to the solution, starting from an initial field $u^{(0)}$ satisfying equations (1), (3) and (4) (but not necessarily

\footnotetext{
${ }^{2}$ One may consider the first kind of boundary condition for the Laplace equation to be Dirichlet's, the second, Neumann's, and the third, Robin's.
} 
equation (2)), and next cyclically correcting the boundary value of the approximation on each of the boundaries of the set of objects. Such a chore is achieved through the introduction of auxiliary fields ${ }^{3}$ (the so-called reflections), defined recursively as the solutions of single-object problems. For the problem at hand, these take the form of the sequences of functions $\left(u_{i}^{(k)}\right)_{k \in \mathbb{N}^{*}}$ and scalars $\left(c_{i}^{(k)}\right)_{k \in \mathbb{N}^{*}}$, with $i$ in $\{1, \ldots, N\}$, such that

$$
\forall i \in\{1, \ldots, N\},\left\{\begin{array}{l}
-\Delta u_{i}^{(1)}=0 \text { in } \Omega \backslash \overline{O_{i}} \\
u_{i}^{(1)}=c_{i}^{(1)}-u^{(0)}-\sum_{j=1}^{i-1} u_{j}^{(1)} \text { on } \partial O_{i}, \\
\int_{\partial O_{i}} \frac{\partial u_{i}^{(1)}}{\partial n}(x) \mathrm{d} S(x)=0, \\
u_{i}^{(1)}=0 \text { on } \partial \Omega
\end{array}\right.
$$

and

$$
\forall k \in \mathbb{N}^{*}, \forall i \in\{1, \ldots, N\},\left\{\begin{array}{l}
-\Delta u_{i}^{(k+1)}=0 \text { in } \Omega \backslash \overline{O_{i}}, \\
u_{i}^{(k+1)}=c_{i}^{(k+1)}-\sum_{j=1}^{i-1} u_{j}^{(k+1)}-\sum_{j=i+1}^{N} u_{j}^{(k)} \text { on } \partial O_{i}, \\
\int_{\partial O_{i}} \frac{\partial u_{i}^{(k+1)}}{\partial \boldsymbol{n}}(x) \mathrm{d} S(x)=0, \\
u_{i}^{(k+1)}=0 \text { on } \partial \Omega .
\end{array}\right.
$$

The approximation of the solution after the $\ell$ th cycle of the method is then defined by summing the restrictions of the reflections to $\Omega \backslash \cup_{j=1}^{N} \overline{O_{j}}$ as follows,

$$
u^{(\ell)}=u^{(0)}+\sum_{k=1}^{\ell} \sum_{i=1}^{N} u_{i}^{(k)} \text { in } \Omega \backslash \cup_{j=1}^{N} \overline{O_{j}} .
$$

We observe that the imposed surface charges are taken into account in the initial approximation $u^{(0)}$ and never subsequently modified by the reflections, which only aim at correcting the boundary values one at a time. The practical construction of such a first approximation does not violate the single-object paradigm of the method, since this field may be obtained as the sum of $N$ solutions to problems of that type by considering each conducting object separately. We also note that the boundary datum for each of the single-object problems defined above depends at a given cycle on quantities computed during both the current cycle and the previous one (or on the initial approximation during the first cycle), which necessarily implies successive computations of their respective solutions, in the spirit of the Gauss-Seidel method to solve linear systems of equations. Hence, we call hereafter this procedure the sequential form of the method of reflections.

Luke [Luk89] showed the unconditional convergence of this version of the method of reflections applied to solve the mobility problem for the Stokes equations (see Subsection 4.2.2). Similar boundary decomposition methods were proposed by Balabane and Tirel [BT97] to solve the Helmholtz equation outside a union of obstacles without trapping rays, and by Coatléven and Joly [CJ12] to effectively solve operator factorized forms of time-harmonic multiple-scattering problems in periodic media.

\subsection{An electrostatic analogue of the resistance problem: the parallel form}

From a mathematical standpoint, the so-called resistance problem of hydrodynamics is a boundary value problem for the Stokes equations with non-homogeneous Dirichlet boundary conditions. Its electrostatic analogue thus amounts to determining the electric potential in a container $\Omega$, its values on the surfaces of $N$ conducting objects $O_{j} \subset \Omega, j$ in $\{1, \ldots, N\}$, being known, that is: given $N$ scalar functions $U_{j}, j$ in $\{1, \ldots, N\}$, respectively defined on $\partial O_{j}, j$ in $\{1, \ldots, N\}$, find the scalar function u satisfying

$$
\begin{aligned}
& -\Delta u=0 \text { in } \Omega \backslash \cup_{j=1}^{N} \overline{O_{j}}, \\
& u=U_{j} \text { on } \partial O_{j}, j=1, \ldots, N .
\end{aligned}
$$

As with the previous example, we assume that the domain

\footnotetext{
${ }^{3}$ The algorithm presented here is a reformulation of the method analysed in [Luk89], as the auxiliary fields do not appear explicitly in the reference.
} 
$\Omega$ is bounded and impose a homogeneous Dirichlet condition on its boundary to complete the problem,

$$
u=0 \text { on } \partial \Omega \text {. }
$$

The second form of the method of reflections hinges on a different construction of the sequence of approximations $\left(u^{(k)}\right)_{k \in \mathbb{N}}$. While the initial approximation $u^{(0)}$ has to satisfy equations (6) and (8) (but not necessarily equation (7)) and the subsequent approximations are still given by the sum (5), the auxiliary fields $\left(u_{i}^{(k)}\right)_{k \in \mathbb{N}^{*}}$, with $i$ in $\{1, \ldots, N\}$, are now defined as the respective solutions to the following single-object problems

$$
\forall i \in\{1, \ldots, N\},\left\{\begin{array}{l}
-\Delta u_{i}^{(1)}=0 \text { in } \Omega \backslash \overline{O_{i}}, \\
u_{i}^{(1)}=U_{i}-u^{(0)} \text { on } \partial O_{i} \\
u_{i}^{(1)}=0 \text { on } \partial \Omega
\end{array}\right.
$$

and

$$
\forall k \in \mathbb{N}^{*}, \forall i \in\{1, \ldots, N\},\left\{\begin{array}{l}
-\Delta u_{i}^{(k+1)}=0 \text { in } \Omega \backslash \overline{O_{i}}, \\
u_{i}^{(k+1)}=-\sum_{j=1}^{i-1} u_{j}^{(k)}-\sum_{j=i+1}^{N} u_{j}^{(k)} \text { on } \partial O_{i}, \\
u_{i}^{(k+1)}=0 \text { on } \partial \Omega .
\end{array}\right.
$$

We observe here that the datum for each of the above problems depends at a given cycle on quantities computed during the previous cycle (and on the boundary data for the first cycle). The solutions of these problems may thus be carried out simultaneously, like in the Jacobi method for the iterative solution of linear systems of equations, leading us to refer to this procedure as the parallel form of the method of reflections.

This version of the method of reflections was devised by Golusin [Gol34] to constructively solve the Dirichlet problem for the Laplace equation in multiply connected circular domains. It appears to be the most commonly found in the literature (see for instance the presentation in subsection 214 of the textbook [Smi64]). Concerning its convergence, Happel and Brenner [HB83] wrote ${ }^{4}$ that: "it must be pointed out that no rigorous proof exists that the iteration scheme converges to the desired solution."

As a matter of fact, a numerical example of divergence for a particular configuration is presented by Ichiki and Brady in [IB01], the method being there used to solve the Stokes resistance problem in the presence of rigid spherical particles in an unbounded domain. The solutions of the single particle problems are numerically approximated by truncated multipole expansions, but the authors conjecture that the observed divergence is unrelated to the order of truncation of the expansions.

In [Tra06], Traytak analysed the method applied to the solution of a Dirichlet problem for the Laplace equation in the unbounded complement of a set of spheres. He obtained necessary and sufficient conditions for its convergence and exhibited simple cases of divergence when the number of spheres is greater than or equal to eight.

More recently, conditional convergence results for the method were derived by Höfer and Velázquez [HV18] for an infinite number of spherical particles, allowing for new proofs of classical homogenization results for the Dirichlet problem for both the Poisson and Stokes equations in perforated domains (see also [Höf18, NS20] which both deal with the mobility problem in a similar context).

This version of the method was also used by Jabin and Otto [JO04] to identify the dilute regime of a cloud of sedimenting particles, in which the particles do not significantly interact and sink as if they were isolated. Additionally, it may be seen that the boundary decomposition technique introduced ${ }^{5}$ by Balabane [Bal04] (see also [GH09, WL13] for practical and numerical applications) for solving a boundary value problem involving the Helmholtz equation in the unbounded complement of a scatterer made of a union of disjoint sub-scatterers is identical to this parallel form of the method. Connections between the principle of this instance of the method and the approach known as the Foldy-Lax $\operatorname{model}^{6}$ [Fol45, Lax51, Lax52] or the generalised Born series technique [Sch85] for the multiple scattering of waves may also be pointed out.

\section{General formulations}

In this section, we summarize and interpret the application of both forms of the method of reflections to the solution of an abstract linear boundary value problem. We want to emphasize that the principle of the method is very general and,

\footnotetext{
${ }^{4}$ The version of the method presented in Chapter 6 of [HB83] differs slightly from the above procedure by focusing on one specific object. The reflections with respect to this object are then computed as in the sequential form of the method, whereas the reflections with respect to the other $N-1$ objects are computed as in the parallel form.

${ }^{5}$ In the same work, a sufficient condition for convergence, depending on the frequency, the diameters and the areas of the sub-scatterers, as well as the respective distances between the sub-scatterers, is established.

${ }^{6} \mathrm{To}$ see this, one can compare the hierarchy of different levels of approximation given in [CH13], ranging from the Born approximation to the Foldy-Lax model, with the sequence of approximations produced by the method of reflections.
} 
because of this generality, the present treatment is necessarily formal. As a consequence, we deliberately refrain from specifying a particular functional framework in order to focus on the algebraic aspects of the method. A rigorous setting is provided in Section 4, with explicit examples given in Subsection 4.2.

We consider a simply connected regular open subset $\Omega$ of $\mathbb{R}^{d}$, and, given a positive integer $N$, a family of arbitrarily numbered "objects" or "holes", which are disjoint subsets $O_{j} \subset \Omega$, with $j$ in $\{1, \ldots, N\}$, in the sense that they are bounded, simply connected, open sets with smooth boundaries such that their closures are non-overlapping. The open set $\Omega \backslash$ $\cup_{j=1}^{N} \overline{O_{j}}$ is then called a perforated domain. We are interested in solving the following non-homogeneous boundary value problem: find a field $u$ satisfying

$$
\begin{aligned}
& \mathscr{L} u=f \text { in } \Omega \backslash \cup_{j=1}^{N} \overline{O_{j}} \\
& \mathscr{B}_{j} u=b_{j}, j=1, \ldots, N,
\end{aligned}
$$

where $\mathscr{L}$ is a linear differential operator, acting on functions defined in $\Omega$, each operator $\mathscr{B}_{j}$, with $j$ in $\{1, \ldots, N\}$, is a linear operator acting, possibly in a non-local manner, on functions defined on the boundary $\partial O_{j}$ of the jth object, and the functions $f$ and $b_{j}$, with $j$ in $\{1, \ldots, N\}$, respectively defined on $\Omega$ and $\partial O_{j}$, with $j$ in $\{1, \ldots, N\}$, are the data of the boundary value problem. In the applications of the method previously recalled, the operator $\mathscr{L}$ is typically of elliptic type, but this is not necessarily the case (see [Bal04] for instance).

Remark 1 Note that the function $f$ is defined over the whole of $\Omega$, while the problem itself is defined over the perforated domain $\Omega \backslash \cup_{j=1}^{N} \overline{O_{j}}$, as customary in problems dealing with homogenisation. In the same way, the functions $b_{j}$, with $j$ in $\{1, \ldots, N\}$, forming the rest of the data are usually related to a single function, globally defined over $\Omega$. We will make such an assumption in Subsection 3.2.

If the domain $\Omega$ is bounded, a homogeneous condition on the boundary $\partial \Omega$ is added to the above system of equations. In the case where it is unbounded, this condition is replaced, or complemented, by one set at infinity. Either way, this boundary condition is denoted

$$
\mathscr{B}_{0} u=0,
$$

and it is assumed that the system (9)-(11) defines a well-posed (in the sense of Hadamard) boundary value problem in an appropriate function space, i.e., there exists a unique solution $u^{*}$ to the problem, which belongs to this space and depends continuously on the data. This implies in particular that the operators $\mathscr{B}_{j}$, with $j$ in $\{1, \ldots, N\}$, verify certain admissibility conditions with respect to the operator $\mathscr{L}$ (see [LM72]).

To solve this boundary value problem using the sequential form of the method of reflections, one begins by computing an initial approximation $u^{(0)}$ satisfying

$$
\left\{\begin{array}{l}
\mathscr{L} u^{(0)}=f \text { in } \Omega, \\
\mathscr{B}_{0} u^{(0)}=0 .
\end{array}\right.
$$

An iterative (or cyclic) phase then follows, in which one solves recursively the single-object problems associated with the reflections given by

$$
\forall i \in\{1, \ldots, N\},\left\{\begin{array}{l}
\mathscr{L}_{i}^{(1)}=0 \text { in } \Omega \backslash \overline{O_{i}}, \\
\mathscr{B}_{i} u_{i}^{(1)}=b_{i}-\mathscr{B}_{i}\left(u^{(0)}+\sum_{j=1}^{i-1} u_{j}^{(1)}\right), \\
\mathscr{B}_{0} u_{i}^{(1)}=0,
\end{array}\right.
$$

and

$$
\forall k \in \mathbb{N}^{*}, \forall i \in\{1, \ldots, N\},\left\{\begin{array}{l}
\mathscr{L} u_{i}^{(k+1)}=0 \text { in } \Omega \backslash \overline{O_{i}}, \\
\mathscr{B}_{i} u_{i}^{(k+1)}=-\mathscr{B}_{i}\left(\sum_{j=1}^{i-1} u_{j}^{(k+1)}+\sum_{j=i+1}^{N} u_{j}^{(k)}\right), \\
\mathscr{B}_{0} u_{i}^{(k+1)}=0,
\end{array}\right.
$$

in order to update the approximation of the solution using the formula

$$
\forall k \in \mathbb{N}, u^{(k+1)}=u^{(k)}+\sum_{i=1}^{N} u_{i}^{(k+1)} \text { in } \Omega \backslash \cup_{j=1}^{N} \overline{O_{j}} .
$$


The approximate solution after $k$ cycles is thus defined by a finite double sum

$$
\forall k \in \mathbb{N}, u^{(k)}=u^{(0)}+\sum_{\ell=1}^{k} \sum_{i=1}^{N} u_{i}^{(\ell)} \text { in } \Omega \backslash \cup_{j=1}^{N} \overline{O_{j}}
$$

We next turn to the parallel version of the method of reflections. In this case, while the initialisation is required to satisfy (12) and the update of the approximate solution is given by (15), the collections of problems to be solved during the cycling phase are

$$
\forall i \in\{1, \ldots, N\},\left\{\begin{array}{l}
\mathscr{L}_{i}^{(1)}=0 \text { in } \Omega \backslash \overline{O_{i}} \\
\mathscr{B}_{i} u_{i}^{(1)}=b_{i}-\mathscr{B}_{i} u^{(0)} \\
\mathscr{B}_{0} u_{i}^{(1)}=0 .
\end{array}\right.
$$

and

$$
\forall k \in \mathbb{N}^{*}, \forall i \in\{1, \ldots, N\},\left\{\begin{array}{l}
\mathscr{L} u_{i}^{(k+1)}=0 \text { in } \Omega \backslash \overline{O_{i}}, \\
\mathscr{B}_{i} u_{i}^{(k+1)}=-\mathscr{B}_{i}\left(\sum_{j=1}^{i-1} u_{j}^{(k)}+\sum_{j=i+1}^{N} u_{j}^{(k)}\right), \\
\mathscr{B}_{0} u_{i}^{(k+1)}=0 .
\end{array}\right.
$$

We observe that both versions of the method of reflections are well-defined as soon as the no-object problem (12) and the respective single-object problems (13) and (14), or (17) and (18), are well-posed. Moreover, the method is said to converge if the sequence of functions defined by (16) has a limit as the number of achieved cycles $k$ tends to infinity.

Remark 2 In some cases, the well-posedness of the single-object problems to be solved during the cyclic phase is a direct consequence of the well-posedness of the many-object boundary value problem one wants to solve with the method. However, considering for instance a non-homogeneous pure Neumann problem for the Laplace operator, one easily sees that the compatibility conditions satisfied by the data of the problem could generally prevent similar conditions to be satisfied by the data of the sub-problems stemming from the boundary decomposition, leading to ill-posed boundary value problems when applying the method. As a consequence, the method of reflections is not applicable to any linear boundary value problem, since the decomposition paradigm on which it is based may not be valid.

\subsection{Algebraic properties of the reflections}

The sequences of reflections $\left(u_{i}^{(k+1)}\right)_{k \in \mathbb{N}}$, with $i$ in $\{1, \ldots, N\}$, constructed by both forms of the method may be viewed as sequences of partial correctors to the sequence of approximate solutions $\left(u^{(k)}\right)_{k \in \mathbb{N}}$ in the following sense.

Lemma 3.1 Suppose boundary value problem (9)-(11) admits a lifting of its boundary data, i.e., there exists a function $g$ defined on $\Omega$ such that $\mathscr{B}_{j} g=b_{j}, j=0, \ldots, N$, and that the $N$ sequences $\left(u_{j}^{(k+1)}\right)_{k \in \mathbb{N}}, j=1, \ldots, N$, produced either by the sequential or by the parallel form of the method of reflections are well defined. Then, one has

$$
\forall k \in \mathbb{N}, \forall i \in\{1, \ldots, N\}, \mathscr{B}_{i}\left(u^{(k)}+\sum_{j=1}^{i} u_{j}^{(k+1)}\right)=\mathscr{B}_{i} g
$$

for the sequential form, and

$$
\forall k \in \mathbb{N}, \forall i \in\{1, \ldots, N\}, \mathscr{B}_{i}\left(u^{(k)}+u_{i}^{(k+1)}\right)=\mathscr{B}_{i} g,
$$

for the parallel form, where the sequence $\left(u^{(k)}\right)_{k \in \mathbb{N}}$ is given by (16).

Proof. Both equalities are easily established by induction. First, for the sequential form, it stems from the linearity of the operators, problem (13), and the definition of $g$ that, for the base case $k=0$ and any integer $i$ in $\{1, \ldots, N\}$,

$$
\mathscr{B}_{i}\left(u^{(0)}+\sum_{j=1}^{i} u_{j}^{(1)}\right)=\mathscr{B}_{i}\left(u^{(0)}+\sum_{j=1}^{i-1} u_{j}^{(1)}+u_{i}^{(1)}\right)=\mathscr{B}_{i}\left(u^{(0)}+\sum_{j=1}^{i-1} u_{j}^{(1)}-u^{(0)}-\sum_{j=1}^{i-1} u_{j}^{(1)}\right)+b_{i}=b_{i}=\mathscr{B}_{i} g \text { on } \partial O_{i} .
$$


Assuming that the equality

$$
\mathscr{B}_{i}\left(u^{(k-1)}+\sum_{j=1}^{i} u_{j}^{(k)}\right)=b_{i},
$$

holds for some positive integer $k$ and any integer $i$ in $\{1, \ldots, N\}$, one has, according to identity (15) and problem (14),

$$
\begin{aligned}
\mathscr{B}_{i}\left(u^{(k)}+\sum_{j=1}^{i} u_{j}^{(k+1)}\right) & =\mathscr{B}_{i}\left(u^{(k-1)}+\sum_{j=1}^{N} u_{j}^{(k)}+\sum_{j=1}^{i} u_{j}^{(k+1)}\right) \\
& =\mathscr{B}_{i}\left(u^{(k-1)}+\sum_{j=1}^{i} u_{j}^{(k)}+\sum_{j=1}^{i} u_{j}^{(k+1)}+\sum_{j=i+1}^{N} u_{j}^{(k)}\right) \\
& =\mathscr{B}_{i}\left(u^{(k-1)}+\sum_{j=1}^{i} u_{j}^{(k)}\right) .
\end{aligned}
$$

Likewise, for the parallel form, one has, for any integer $i$ in $\{1, \ldots, N\}$,

$$
\mathscr{B}_{i}\left(u^{(0)}+u_{i}^{(1)}\right)=\mathscr{B}_{i} u^{(0)}+b_{i}-\mathscr{B}_{i} u^{(0)}=b_{i},
$$

using the linearity of the operators, problem (17), the definition of $g$, and assuming that

$$
\mathscr{B}_{i}\left(u^{(k-1)}+u_{i}^{(k)}\right)=\mathscr{B}_{i} g
$$

is satisfied for some integer positive $k$ and any integer $i$ in $\{1, \ldots, N\}$, one finds that

$$
\begin{aligned}
\mathscr{B}_{i}\left(u^{(k)}+u_{i}^{(k+1)}\right) & =\mathscr{B}_{i}\left(u^{(k-1)}+\sum_{j=1}^{N} u_{j}^{(k)}+u_{i}^{(k+1)}\right) \\
& =\mathscr{B}_{i}\left(u^{(k-1)}+\sum_{j=1}^{N} u_{j}^{(k)}-\sum_{j=1}^{i-1} u_{j}^{(k)}-\sum_{j=i+1}^{N} u_{j}^{(k)}\right) \\
& =\mathscr{B}_{i}\left(u^{(k-1)}+u_{i}^{(k)}\right),
\end{aligned}
$$

using identity (15) and problem (18), which ends the proof.

Remark 3 An analogue of formula (20) was derived by Höfer and Velázquez (see equation (28) in [HV18]) in the case of the screened Poisson equation with homogeneous Dirichlet boundary conditions and an infinite number of objects.

To get a result on the possible limit of sequence (16), a minimal functional framework needs to be specified. Namely, we assume from now on that problem (9)-(11) is set in a Banach space.

Proposition 3.2 If the sequence of functions, defined by (16) and produced by either form of the method of reflections, converges (in the sense of an absolutely convergent series), then it is to the solution $u^{*}$ to boundary value problem (9)-(11).

Proof. From problems (12), (13) and (14) (resp. (17) and (18)), and formula (16), it is easily seen that any element of the sequence $\left(u^{(k)}\right)_{k \in \mathbb{N}}$, produced by the sequential (resp. parallel) form of the method of reflections, satisfies

$$
\left\{\begin{array}{l}
\mathscr{L} u^{(k)}=f \text { in } \Omega \backslash \cup_{j=1}^{N} \overline{O_{j}}, \\
\mathscr{B}_{0} u^{(k)}=0,
\end{array}\right.
$$

and thus, by continuity, so does the limit of this sequence. Next, using that the convergence implies that

$$
\forall i \in\{1, \ldots, N\}, \lim _{k \rightarrow+\infty} u_{i}^{(k)}=0,
$$

it follows from passing to the limit in $k$ in (19) (resp. (20)) that

$$
\forall i \in\{1, \ldots, N\}, \lim _{k \rightarrow+\infty} \mathscr{B}_{i} u^{(k)}=b_{i},
$$

so that the limit of the sequence indeed solves problem (9)-(11). 
Remark 4 For any integer $i$ in $\{1, \ldots, N\}$, the partial sum $\sum_{\ell=0}^{k} u_{i}^{(\ell)}$ represents the contribution of the ith object to the approximate solution after $k$ cycles. As the convergence of the method entails the convergence of the sequences of partial sums, the method of reflections leads to a constructive and natural (i.e., with respect to the object boundaries) decomposition result for the solution to the boundary value problem, similar to the one obtained in Theorem 1 in [Bal04]. The existence of such a decomposition, which can be viewed as a specific application of the superposition principle, suggests that the method is best combined with numerical methods based on an integral representation formula. The basic ingredient in this formalism is the so-called Green function of the governing linear differential operator, which also allows to compute the initial approximation as a volume potential (also called a Newton potential in the case of the Laplace equation) and the subsequent reflections as surface (single-layer and/or double-layer) potentials.

\subsection{Projection setting}

Following Luke [Luk89] and Höfer and Velázquez [HV18, Höf18], we propose an interpretation of the method of reflections in terms of projection operators. In order to properly define such operators, the problem to be solved and the various subproblems considered by the method, all defined on different subsets of $\Omega$, first need to be extended to the whole of $\Omega$, by the adjunction of problems defined in the interior of the objects and the use of transmission conditions across the boundaries of the objects. In what follows, we will abuse the notation by denoting in the same way the functions defined on $\Omega \backslash \cup_{j=1}^{N} \overline{O_{j}}$ (the reflection $u_{i}^{(k)}$, the approximation $u^{(k)}$ and the solution $u^{*}$ ) and their respective extensions.

Let $H$ be a function space defined over the set $\Omega$ in which the resulting exterior-interior transmission problems and the initialisation problem (12) are well-posed. For any function $u$ in $H$, consider the functions $v$ and $w$ in $H$, such that $u=v+w$, with $w$ satisfying the following problem in the exterior of the objects,

$$
\left\{\begin{array}{l}
\mathscr{L} w=\mathscr{L} u \text { in } \Omega \backslash \cup_{j=1}^{N} \overline{O_{j}}, \\
\mathscr{B}_{j} w=0, j=1, \ldots, N, \\
\mathscr{B}_{0} w=0,
\end{array}\right.
$$

the following equation in the interior of the objects,

$$
\mathscr{L} w=0 \text { in } \cup_{j=1}^{N} O_{j},
$$

and some chosen transmission conditions across the boundaries of the objects, which depend on the differential operator $\mathscr{L}$ and are such that a solution to the resulting exterior-interior transmission problem (21)-(22) exists and is uniquely defined in $H$. The functions $v$ and $w$ defined in this way are unique and depend continuously on $u$. It then follows that the mappings $u \mapsto v$ and $u \mapsto w$ from $H$ to $H$ are bounded. Since they also are idempotent by construction, they are continuous linear projection operators, with respective closed ranges $V$ and $M$ such that

$$
H=V \oplus M .
$$

In what follows, we shall assume that boundary value problem (9)-(11) admits a lifting of its boundary data, i.e., there exists a function $g$ defined on $\Omega$ such that $\mathscr{B}_{i} g=b_{i}$ on $\partial O_{i}$, for any integer $i$ in $\{0, \ldots, N\}$.

Proposition 3.3 Suppose that problem (9)-(11) admits a lifting $g$ in $H$ of its boundary data, that there exists an initial approximation $u^{(0)}$ in $H$ satisfying (12), and that the multiple-object exterior-interior transmission problem (21)-(22) is well-posed in $H$ for any choice of $u$ in $H$, so that, in particular, decomposition (23) holds. Then, the solution to problem (9)-(11) is given by the restriction to $\Omega \backslash \cup_{j=1}^{N} \overline{O_{j}}$ of

$$
u^{*}=P_{V} g+P_{M} u^{(0)}
$$

where $P_{V}$ denotes the projection from $H$ onto $V$ along $M$, and $P_{M}$ denotes the projection from $H$ onto $M$ along $V$.

Proof. Owing to the definitions of $g$ and $u^{(0)}$, one can rewrite problem (9)-(11) as

$$
\left\{\begin{array}{l}
\mathscr{L} u=\mathscr{L}^{(0)} \text { in } \Omega \backslash \cup_{j=1}^{N} \overline{O_{j}}, \\
\mathscr{B}_{j} u=\mathscr{B}_{j} g, j=1, \ldots, N, \\
\mathscr{B}_{0} u=\mathscr{B}_{0} g .
\end{array}\right.
$$


Observing that $P_{V} g$ and $P_{M} u^{(0)}$ satisfy the following systems in the exterior of the objects

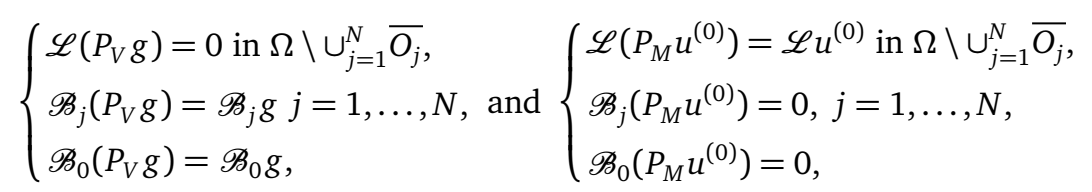

we conclude using the linearity of the problems.

Remark 5 The function $u^{*}$ introduced in Proposition 3.3 is an extension to the whole of domain $\Omega$ of the solution of the problem set in the perforated domain $\Omega \backslash \cup_{j=1}^{N} \overline{O_{j}}$, satisfying

$$
\mathscr{L} u^{*}=\mathscr{L} g \text { in } \cup_{j=1}^{N} O_{j} .
$$

For any integer $i$ in $\{1, \ldots, N\}$, considering well-posed single-object exterior-interior transmission problems in place of (21)-(22), made up of systems of the form

$$
\forall i \in\{1, \ldots, N\},\left\{\begin{array}{l}
\mathscr{L} w=\mathscr{L} u \text { in } \Omega \backslash \overline{O_{i}}, \\
\mathscr{B}_{i} w=0, \\
\mathscr{B}_{0} w=0,
\end{array} \text { and } \mathscr{L} w=0 \text { in } O_{i},\right.
$$

completed by a transmission condition across the boundary $\partial O_{i}$ similar to those previously chosen for the multiple-object problem, one can similarly introduce the projection operators $P_{V_{i}}$ and $P_{M_{i}}$, with respective complementary closed ranges $V_{i}$ and $M_{i}$ in $H$. It is then clear that

$$
M=\cap_{j=1}^{N} M_{j}
$$

As was done in Proposition 3.3 for the solution to problem (9)-(11), the reflections can be shown to be the restrictions of some quantities involving these projection operators. More precisely, we have the following result, whose proof is left to the reader.

Proposition 3.4 Suppose that problem (9)-(11) admits a lifting $g$ in $H$ of its boundary data, that there exists an initial approximation $u^{(0)}$ in $H$ satisfying (12), and that the single-object exterior-interior transmission problems (24) are wellposed in $H$ for any choice of $u$ in $H$. Then, for any integer $i$ in $\{1, \ldots, N\}$, the reflection $u_{i}^{(k)}$ is given by the restriction to $\Omega \backslash \overline{O_{i}}$ of

$$
\begin{gathered}
-P_{V_{i}}\left(u^{(0)}+\sum_{j=1}^{i-1} u_{j}^{(1)}-g\right)\left(\text { resp. }-P_{V_{i}}\left(u^{(0)}-g\right) \text { ) if } k=1,\right. \\
-P_{V_{i}}\left(\sum_{j=1}^{i-1} u_{j}^{(k)}+\sum_{j=i+1}^{N} u_{j}^{(k-1)}\right) \text { (resp. }-P_{V_{i}}\left(\sum_{j=1}^{i-1} u_{j}^{(k-1)}+\sum_{j=i+1}^{N} u_{j}^{(k-1)}\right) \text { ) if } k>1,
\end{gathered}
$$

for the sequential (resp. parallel) version of the method of reflections, where $P_{V_{i}}$ denotes the projection from $H$ onto $V_{i}$ along $M_{i}$.

We finally derive some relations needed for the forthcoming analysis of the method of reflections, starting with the sequential version.

Proposition 3.5 Under the assumptions and notations of Proposition 3.4, the following recurrence formula holds for the sequence of approximations produced by the sequential form of the method of reflections,

$$
\forall k \in \mathbb{N}, u^{(k+1)}-g=E_{N}\left(u^{(k)}-g\right),
$$

where $E_{N}=\left(I d_{H}-P_{V_{N}}\right) \ldots\left(I d_{H}-P_{V_{1}}\right)$, with $I d_{H}$ the identity operator on $H$.

Proof. Let us denote $E_{0}=I d_{H}, E_{i}=\left(I d_{H}-P_{V_{i}}\right) E_{i-1}$, with $i$ in $\{1, \ldots, N\}$. We shall first prove by induction that

$$
\forall k \in \mathbb{N}, \forall i \in\{1, \ldots, N\}, u_{i}^{(k+1)}=-P_{V_{i}} E_{i-1}\left(u^{(k)}-g\right) .
$$


First, it follows from (19) that

$$
\forall k \in \mathbb{N}, \forall i \in\{1, \ldots, N\}, \mathscr{B}_{i}\left(u_{i}^{(k+1)}\right)=-\mathscr{B}_{i}\left(u^{(k)}-g+\sum_{j=1}^{i-1} u_{j}^{(k+1)}\right),
$$

which, due to the definition of the projection operator $P_{V_{i}}$ and to the extension of problem (14), translates into

$$
\forall k \in \mathbb{N}, \forall i \in\{1, \ldots, N\}, u_{i}^{(k+1)}=-P_{V_{i}}\left(u^{(k)}-g+\sum_{j=1}^{i-1} u_{j}^{(k+1)}\right) .
$$

For $i=1$, this identity is simply

$$
\forall k \in \mathbb{N}, u_{1}^{(k+1)}=-P_{V_{1}}\left(u^{(k)}-g\right) .
$$

Next, assume that

$$
\forall k \in \mathbb{N}, \forall i \in\{1, \ldots, N-1\}, u_{i}^{(k+1)}=-P_{V_{i}} E_{i-1}\left(u^{(k)}-g\right) .
$$

Then, one has

$$
u_{i+1}^{(k+1)}=-P_{V_{i+1}}\left(u^{(k)}-g+\sum_{j=1}^{i} u_{j}^{(k+1)}\right)=-P_{V_{i+1}}\left(I d-\sum_{j=1}^{i} P_{V_{j}} E_{j-1}\right)\left(u^{(k)}-g\right)=-P_{V_{i+1}} E_{i}\left(u^{(k)}-g\right),
$$

which ends the induction. Using definition (15), one finally reaches

$$
u^{(k+1)}-g=u^{(k)}-g+\sum_{i=1}^{N} u_{i}^{(k+1)}=\left(I d-\sum_{i=1}^{N} P_{V_{i}} E_{i-1}\right)\left(u^{(k)}-g\right)=E_{N}\left(u^{(k)}-g\right) .
$$

An analogous result holds for the parallel version of the method.

Proposition 3.6 Under the assumptions and notations of Proposition 3.4, the following recurrence formula holds for the sequence of approximations produced by the parallel form of the method of reflections,

$$
\forall k \in \mathbb{N}, u^{(k+1)}-g=\left(I d-\sum_{i=1}^{N} P_{V_{i}}\right)\left(u^{(k)}-g\right)
$$

Proof. Owing to (20), one has

$$
\forall k \in \mathbb{N}, \forall i \in\{1, \ldots, N\}, \mathscr{B}_{i} u_{i}^{(k+1)}=-\mathscr{B}_{i}\left(u^{(k)}-g\right),
$$

which gives, due to the definition of the projection operator $P_{V_{i}}$ and the extension of (18),

$$
\forall k \in \mathbb{N}, \forall i \in\{1, \ldots, N\}, u_{i}^{(k+1)}=-P_{V_{i}}\left(u^{(k)}-g\right) .
$$

Substituting into (15), one reaches

$$
\forall k \in \mathbb{N}, u^{(k+1)}-g=u^{(k)}-g+\sum_{i=1}^{N} u_{i}^{(k+1)}=u^{(k)}-g-\sum_{i=1}^{N} P_{V_{i}}\left(u^{(k)}-g\right),
$$

yielding (26).

\section{Convergence analysis in a Hilbert space setting}

We now flesh out the structure of the previous mathematical setting by making a number of additional assumptions. We suppose that the function space $H$ is a real Hilbert space and that the problem to solve can be written under an equivalent weak formulation, involving a continuous bilinear form $a(\cdot, \cdot)$ from $H \times H$ to $\mathbb{R}$ satisfying inf-sup conditions 
which ensure the well-posedness of the problem. In this context, the projection operators $P_{M_{i}}$, with $i$ in $\{1, \ldots, N\}$, previously introduced can be defined by

$$
\forall u \in H, \forall v \in M_{i}, a\left(P_{M_{i}} u, v\right)=a(u, v),
$$

(see [XZ02] for instance), and the method of reflections is said to be convergent in $H$ if the sequence $\left(u^{(k)}\right)_{k \in \mathbb{N}}$ tends to $u^{*}$ with respect to the norm on $H$. In view of the respective formulas derived in Propositions 3.5 and 3.6, it is then clear that the convergence of the method is tied to the behaviour of the fixed-point iteration of a bounded linear mapping $T$ from $H$ to itself, where $T=E_{N}=\left(I d_{H}-P_{V_{N}}\right) \ldots\left(I d_{H}-P_{V_{1}}\right)$ for the sequential form of the method of reflections, or $T=I d_{H}-\sum_{i=1}^{N} P_{V_{i}}$ for the parallel one.

\subsection{The orthogonal case: theoretical results}

For some boundary value problems of the form (9)-(11), notably when the operator $\mathscr{L}$ is of elliptic type and the boundary conditions are essential, convenient choices of the Hilbert space $H$ make the bilinear form $a(\cdot, \cdot)$ introduced above an equivalent inner product on $H$. This results in the linear mappings $P_{M_{i}}$, with $i$ in $\{1, \ldots, N\}$, satisfying (27), being orthogonal projection operators. This observation is at the origin of the method of orthogonal projections, developed by Vishik [Vis49] in connection with previous works by Zaremba [Zar27] and Weyl [Wey40], and adapted by Hruslov for a problem for an elliptic operator set in a perforated domain [Hru72].

Under the above assumptions, for any integer $i$ in $\{1, \ldots, N\}$, the subspace $V_{i}=M_{i}{ }^{\perp}$ is the orthogonal complement of $M_{i}$ in $H$ and $P_{V_{i}}=I d-P_{M_{i}}$ is also an orthogonal projection operator. Likewise, we have that $V=M^{\perp}$, so that

$$
V=\left(\cap_{j=1}^{N} M_{j}\right)^{\perp}=\overline{\sum_{j=1}^{N} M_{j}^{\perp}}=\overline{\sum_{j=1}^{N} V_{j}} .
$$

We will now address the convergence of both forms of the method within this framework.

\subsubsection{Sequential form}

In the case of orthogonal projection operators, the sequential form of the method of reflections is closely related to the method of alternating (or cyclic) projections (MAP for short), which is a simple iterative procedure for determining the orthogonal projection of an element onto an intersection of closed (linear) subspaces $M_{1}, \ldots, M_{N}$ of a Hilbert space $H$ using a sequence of orthogonal projections onto those subspaces. It has numerous applications (the interested reader may check the review by Deutsch [Deu92] for details) and its design relies on the pointwise convergence result, first proved by von Neumann in 1933 (but not published until 1949) in the $N=2$ case while working on the theory of operators [vNe49] and later generalised by Halperin [Hal62] to any integer $N \geq 2$, stating that one has

$$
\forall v \in H, \lim _{k \rightarrow+\infty}\left\|\left(P_{M_{N}} P_{M_{N-1}} \ldots P_{M_{1}}\right)^{k} v-P_{\cap_{j=1}^{N} M_{j}} v\right\|_{H}=0 .
$$

Consequently, the computation of the orthogonal projection of a point $v$ in $H$ onto $\cap_{j=1}^{N} M_{j}$ by the MAP consists in building the sequence $\left(w^{(k)}\right)_{k \in \mathbb{N}}$ defined by

$$
w^{(0)}=v \text { and, } \forall k \in \mathbb{N}, w^{(k+1)}=P_{M_{N}} \ldots P_{M_{1}} w^{(k)},
$$

that is, by recursively applying the orthogonal projections to the current iterate. It follows that recurrence relation (25) for the sequential version of the method of reflections is simply a particular application of the MAP. Thus, using that $P_{V}+P_{M}=I d_{H}$ and that, for any integer $i$ in $\{1, \ldots, N\}, P_{V_{i}} P_{M}=0$, one can write that

$$
\forall k \in \mathbb{N}^{*}, u^{(k)}=\left(E_{N}\right)^{k}\left(u^{(0)}-P_{V} g\right)+P_{V} g,
$$

with $E_{N}=\left(I d_{H}-P_{V_{N}}\right) \ldots\left(I d_{H}-P_{V_{1}}\right)=P_{M_{N}} P_{M_{N-1}} \ldots P_{M_{1}}$, so that passing to the limit yields

$$
\lim _{k \rightarrow+\infty} u^{(k)}=P_{M}\left(u^{(0)}-P_{V} g\right)+P_{V} g=P_{M}\left(u^{(0)}\right)+P_{V} g=u^{*} \text { in } H .
$$

The unconditional convergence of the method ensues and we have proved the following result.

Theorem 4.1 Under the assumptions and notations of Proposition 3.4, suppose that the projection operators $P_{V_{i}}$, with $i$ in $\{1, \ldots, N\}$, are orthogonal. Then, the sequence of approximations $\left(u^{(k)}\right)_{k \in \mathbb{N}}$ generated by the sequential form of the method of reflections converges to the solution $u^{*}$ in $H$. 


\subsubsection{Parallel form}

The fact that the projection operators are orthogonal does not allow one to conclude that the parallel version of the method of reflections is convergent as it is the case for its sequential form. Nevertheless, if one is able to show that the operator $T=I d_{H}-\sum_{j=1}^{N} P_{V_{j}}$ is nonexpansive ${ }^{7}$ and asymptotically regular ${ }^{8}$, it is known (see Corollary 2.3 in $\left[\mathrm{BDH}^{+} 03\right]$ ) that, for any $u$ in $H$, the sequence $\left(T^{k} u\right)_{k \in \mathbb{N}}$ converges to $P_{\mathrm{Fix} T} u$ in $H$, where $P_{\mathrm{Fix} T}$ is the orthogonal projector on the fixed point set for $T$, and that ${ }^{9}$ Fix $T=M$. Two major drawbacks of such a result is that the imposed conditions on the operator $T$ are often not readily checkable and that it does not hold in a nonorthogonal context. As a consequence, geometric conditions on the objects and their respective positions ensuring the summability of the series defined by (16) are generally preferred (see for instance [Tra06] or [Bal04] in a nonorthogonal framework).

Nevertheless, one maay obtain an unconditionally convergent and properly parallel method by modifying the algorithm in such a way that the resulting recurrence formula reads

$$
\forall k \in \mathbb{N}, u^{(k+1)}-g=\left(I d-\frac{1}{N} \sum_{j=1}^{N} P_{V_{j}}\right)\left(u^{(k)}-g\right)=\left(\frac{1}{N} \sum_{j=1}^{N}\left(I d-P_{V_{j}}\right)\right)\left(u^{(k)}-g\right) .
$$

This alteration can be interpreted as a relaxation of the recurrence relation (26) of the form

$$
\forall k \in \mathbb{N}, u^{(k+1)}-g=v\left(I d-\sum_{j=1}^{N} P_{V_{j}}\right)\left(u^{(k)}-g\right)+(1-v)\left(u^{(k)}-g\right),
$$

with relaxation factor $v=\frac{1}{N}$, yielding

$$
\forall k \in \mathbb{N}, u^{(k)}-g=\left(\frac{1}{N} \sum_{j=1}^{N}\left(I d-P_{V_{j}}\right)\right)^{k}\left(u^{(0)}-g\right) .
$$

It is then a well-known fact, since the work of Cimmino [Cim38] on the method of averaged (or simultaneous) projections (an iterative method to solve linear systems of equation based on a geometrical approach), and its extension by Auslender [Aus76], that, for any closed (linear) subspaces $M_{1}, \ldots, M_{N}$ of a Hilbert space $H$, one has the pointwise convergence result ${ }^{10}$

$$
\forall v \in H, \lim _{k \rightarrow+\infty}\left\|\left(\frac{1}{N} \sum_{j=1}^{N} P_{M_{j}}\right)^{k} v-P_{\cap_{j=1}^{N} M_{j}} v\right\|_{H}=0 .
$$

The convergence of the method then follows from the same arguments as in the previous subsection.

Theorem 4.2 Under the assumptions and notations of Proposition 3.4, suppose that the projection operators $P_{V_{i}}$, with $i$ in $\{1, \ldots, N\}$, are orthogonal. Then, the sequence of approximations $\left(u^{(k)}\right)_{k \in \mathbb{N}}$ generated by the averaged parallel version of the parallel form of the method of reflections converges to the solution $u^{*}$ in $H$.

In terms of induced practical changes, the update formula for the approximation of the solution (see equation (15)) becomes

$$
\forall k \in \mathbb{N}, u^{(k+1)}=u^{(k)}+\frac{1}{N} \sum_{j=1}^{N} u_{j}^{(k+1)} \text { in } \Omega \backslash \cup_{j=1}^{N} \overline{O_{j}},
$$

\footnotetext{
${ }^{7}$ The mapping $T$ is said to be nonexpansive on the normed space $H$ if it is a function from $H$ to itself such that

$$
\forall(u, v) \in H^{2},\|T u-T v\|_{H} \leq\|u-v\|_{H} .
$$

${ }^{8}$ The mapping $T$ is said to be asymptotically regular on the normed space $H$ if it is a function from $H$ to itself such that

$$
\forall u \in H, \lim _{k \rightarrow+\infty}\left\|T^{k+1} u-T^{k} u\right\|_{H}=0 .
$$

${ }^{9}$ Indeed, one has trivially that $M \subset$ Fix $T$, while the converse follows from the idempotence and the self-adjointness of the orthogonal projectors.

${ }^{10}$ This result remains valid for the more general sum $\sum_{j=1}^{N} \gamma_{j} P_{M_{j}}$, where the scalars $\gamma_{i}$, with $i$ in $\{1, \ldots, N\}$, are the weights of a convex combination (that is, such that, $\forall i \in\{1, \ldots, N\}, \gamma_{i}>0$, and $\sum_{j=1}^{n} \gamma_{j}=1$ ).
} 
the reflection $u_{i}^{(k+1)}$ being obtained by solving sub-problem (18) in which the second equation is replaced by

$$
\mathscr{B}_{i}\left(u_{i}^{(k+1)}\right)=-\mathscr{B}_{i}\left(\frac{1}{N}\left(\sum_{j=1}^{i-1} u_{j}^{(k)}+\sum_{j=i+1}^{N} u_{j}^{(k)}\right)-\left(1-\frac{1}{N}\right) u_{i}^{(k)}\right),
$$

confirming that the modification may be viewed as a simple relaxation method applied to the parallel form of the method of reflections. One may also remark that the interpretation of the auxiliary fields as "reflections" remains, since it can be shown that equality (20) still holds. We thus refer to this variant as the averaged parallel form of the method of reflections.

\subsubsection{Rate of convergence}

Let us now deal with the rate of convergence of the method by recalling the following dichotomy property for the MAP (see Theorem 6.4 in [DH10] and also Theorem 1.4 in [BDH09] for the case $N=2$ ):

- If $\sum_{j=1}^{N} M_{j}{ }^{\perp}$ is closed, the sequence $\left(\left(P_{M_{N}} P_{M_{N-1}} \ldots P_{M_{1}}\right)^{k}\right)_{k \in \mathbb{N}}$ converges to $P_{\cap_{j=1}^{N} M_{j}}$ linearly ${ }^{11}$, that is, there exist constants $C>0$ and $\alpha \in[0,1)$ such that

$$
\forall k \in \mathbb{N},\left\|\left(P_{M_{N}} P_{M_{N-1}} \ldots P_{M_{1}}\right)^{k}-P_{\cap_{j=1}^{N} M_{j}}\right\|_{\mathscr{L}(H)} \leq C \alpha^{k} .
$$

- If $\sum_{j=1}^{N} M_{j}{ }^{\perp}$ is not closed, the sequence $\left(\left(P_{M_{N}} P_{M_{N-1}} \ldots P_{M_{1}}\right)^{k}\right)_{k \in \mathbb{N}}$ converges to $P_{\cap_{j=1}^{N} M_{j}}$ arbitrarily slowly, that is,

(i) the sequence $\left(\left(P_{M_{N}} P_{M_{N-1}} \ldots P_{M_{1}}\right)^{k}\right)_{k \in \mathbb{N}}$ converges pointwise to $P_{\bigcap_{j=1}^{N} M_{j}}$,

(ii) for each real-valued function $\phi$ on the positive integers that converges to 0 , there exists a point $v$ in $H$ such that

$$
\forall k \in \mathbb{N},\left\|\left(P_{M_{N}} P_{M_{N-1}} \ldots P_{M_{1}}\right)^{k} v-P_{\bigcap_{j=1}^{N} M_{j}} v\right\|_{H} \geq \phi(k) .
$$

The implication of this result for the sequential form of the method of reflections is that it converges linearly as soon as the sum $\sum_{j=1}^{N} V_{j}$ is closed, that is if $V=\sum_{j=1}^{N} V_{j}$. Equivalent conditions, based on the notion of angle ${ }^{12}$ between subspaces, can be found in the literature ${ }^{13}$, and it is worth noting that the linear convergence of the (sequential version of the) method of reflections was established in [Luk89] using conditions on any two sub-collections of the set of involved subspaces and the notion of gap between these.

Note that, in the case of a linear convergence of the MAP, error bounds can be derived, leading to estimates for the sequential for of the method of reflections. Let us gather here some of the existing results on this topic. When $N=2$, one has $\left\|\left(P_{M_{2}} P_{M_{1}}\right)^{k}-P_{M_{1} \cap M_{2}}\right\|_{\mathscr{L}(H)} \leq c\left(M_{1}, M_{2}\right)^{2 k-1}$, where $c\left(M_{1}, M_{2}\right)$ denotes the cosine of the angle between the subspaces $M_{1}$ and $M_{2}$. This result is due to Aronszajn (see [Aro50, Section 12]) and has been rediscovered several times. It is also sharp (see [KW88, Theorem 2]). For $N \geq 3$, upper bounds were given by Smith, Solomon and Wagner [SSW77, Theorem 2.2], Kayalar and Weinert [KW88, Theorem 3], and also Deutsch and Hundal [DH97, Theorem 2.7]. In this case however, note that any error bound depending only on the angles between the various subspaces involved can never

\footnotetext{
${ }^{11}$ Some authors say the sequence converges uniformly (see [BGM12]).

${ }^{12}$ According to the definition of Friedrichs [Fri37], if $M_{i}$ and $M_{j}$ are closed subspaces in a Hilbert space $H$, the angle between $M_{i}$ and $M_{j}$ is the angle in $\left[0, \frac{\pi}{2}\right]$ whose cosine is defined by

$$
c\left(M_{i}, M_{j}\right)=\sup \left\{|\langle x, y\rangle| \mid x \in M_{i} \cap\left(M_{i} \cap M_{j}\right)^{\perp},\|x\| \leq 1, y \in M_{j} \cap\left(M_{i} \cap M_{j}\right)^{\perp},\|y\| \leq 1\right\} .
$$

Another notion is that of the minimal angle between $M_{i}$ and $M_{j}$, given by Dixmier in [Dix49], which is the angle in [0, $\frac{\pi}{2}$ ] whose cosine is defined by

$$
c_{0}\left(M_{i}, M_{j}\right)=\sup \left\{|\langle x, y\rangle| \mid x \in M_{i},\|x\| \leq 1, y \in M_{j},\|y\| \leq 1\right\} .
$$

These two definitions are different if $M_{i} \cap M_{j} \neq\{0\}$, and they both coincide with (different) principal angles (as introduced by Jordan [Jor75]) if $H=\mathbb{C}^{n}$.

${ }^{13}$ For $N=2$, it is known (see [Deu85] for instance) that $c\left(M_{1}, M_{2}\right)<1$, where $c\left(M_{1}, M_{2}\right)$ denotes the cosine of the angle between the subspaces $M_{1}$ and $M_{2}$, if and only if $M_{1}+M_{2}$ is closed, if and only if $M_{1}{ }^{\perp}+M_{2} \perp$ is closed, if and only if $\left(M_{1} \cap\left(M_{1} \cap M_{2}\right)^{\perp}\right)+\left(M_{2} \cap\left(M_{1} \cap M_{2}\right)^{\perp}\right)$ is closed. For $N \geq 3$, a generalization of the Friedrichs angle to several subspaces is introduced in [BGM12] and it is shown in the same paper that the method converges linearly if and only if $c\left(M_{1}, \ldots, M_{N}\right)<1$, which is a weaker condition (see Example 4.5 in [BGM12]) than the sufficient one, based on Theorems 2.1 in [DH97] and 4.1 in [BGM12], that one of the cosines $c_{i j}=c_{0}\left(M_{i} \cap\left(\cap_{\ell=1}^{N} M_{\ell}\right)^{\perp}, M_{j} \cap\left(\cap_{\ell=1}^{N} M_{\ell}\right)^{\perp}\right)$ of the Dixmier angles involving two subspaces $M_{i}$ and $M_{j}$ is strictly less than one.
} 
be sharp [DH97, Example 3.7]. More recently, using the link between the MAP and the SSC methods, Xu and Zikatanov [XZ02] obtained, under the assumption that the sum $\sum_{j=1}^{N} M_{j}{ }^{\perp}$ is closed, the following equality

$$
\left\|P_{M_{N}} P_{M_{N-1}} \ldots P_{M_{1}}-P_{\cap_{j=1}^{N} M_{j}}\right\|_{\mathscr{L}(H)}^{2}=\frac{c_{0}}{1+c_{0}}
$$

where

$$
c_{0}=\sup _{\substack{v \in V \\\|v\|_{H}=1}} \inf _{\substack{\left(v_{1}, \ldots, v_{N}\right) \in M_{1}^{\perp} \times \cdots \times M_{N}{ }^{\perp} \\ \sum_{k=1}^{N} v_{k}=v}} \sum_{j=1}^{N}\left\|\left(I d_{H}-P_{M_{j}}\right) \sum_{k=j+1}^{N} v_{k}\right\|_{H}{ }^{2} .
$$

We may add that various ways of accelerating the MAP, using relaxation or symmetrization for instance, have been proposed and studied (see $\left[\mathrm{BDH}^{+} 03\right]$ and the references therein), and could be directly used on the sequential version of the method of reflections.

Finally, because simultaneous projections correspond to alternating projections in the adequate product space (see [Pie84]), variants of the above results exist for the method of simultaneous projections, as a consequence of results in [BB96, BDH09], and thus apply to the averaged parallel version of the method of reflections.

\subsection{The orthogonal case: practical examples}

It follows from the previous results that the orthogonality of the projection operators associated with the boundary value problem to solve is a sufficient condition for the convergence of the method, either in its sequential form or in an averaged version of its parallel form. As an application, we prove this property for problems in which the main differential operator is of elliptic type. More precisely, our attention is focused on examples that involve the Laplace and the Stokes operators. For each of them, we describe the extended boundary value problems and characterise the functional setting involved in the definition of the projection operators. In addition to proving the orthogonality of the projectors, we also show that the rate of convergence is linear, by establishing that the sum $\sum_{j=1}^{N} V_{j}$ is closed.

\subsubsection{The Laplace and Poisson equations}

In what follows, it is assumed that the domain $\Omega$ is a bounded, simply connected, open set of $\mathbb{R}^{d}$, with boundary $\partial \Omega$, containing $N$ simply connected open subdomains $O_{j}$, with respective boundaries $\partial O_{j}$, with $j$ in $\{1, \ldots, N\}$. All the boundaries are supposed to be sufficiently smooth, twice continuously differentiable for instance.

We deal with boundary value problems of the form considered in Section 3, for which the operator $\mathscr{L}$ is the negative Laplace operator and $\mathscr{B}_{0}$ is the trace operator on $\partial \Omega$. These choices respectively correspond to the Poisson equation if the datum $f$ is non-zero, else to the Laplace equation, with a homogeneous Dirichlet boundary condition on $\partial \Omega$. They have to be complemented with some conditions on the boundaries $\partial O_{j}$, with $j$ in $\{1, \ldots, N\}$, of the objects, for which different choices are possible.

Poisson problem with Dirichlet boundary conditions. As in the boundary value problem appearing in Subsection 2.2, we consider Dirichlet-type boundary conditions, meaning that, for any integer $i$ in $\{1, \ldots, N\}$, the boundary operator $\mathscr{B}_{i}$ is the trace operator on $\partial O_{i}$. If the datum $f$ belongs to $H^{-1}(\Omega)$ and the boundary data $b_{j}$, with $j$ in $\{1, \ldots, N\}$, are respectively in $H^{1 / 2}\left(\partial O_{j}\right)$, one may show, using the Lax-Milgram lemma, that the problem is well-posed in the space $\left\{u \in H^{1}\left(\Omega \backslash \cup_{j=1}^{N} \overline{O_{j}}\right) \mid u_{\mid \partial \Omega}=0\right\}$.

To extend the problem to the interior of the objects, we impose the following transmission conditions across the object boundaries

$$
\left[u^{*}\right]=0 \text { across } \partial O_{j}, j=1, \ldots, N,
$$

where the brackets $[\cdot]$ denote the jump across the considered hypersurface. We may then set the exterior-interior transmission problem in the space $H=H_{0}^{1}(\Omega)$, and it follows from the surjectivity of the trace operator that there exists a lifting $g$ in $H$ such that $g=b_{j}$ on $\partial O_{j}$, for any integer $j$ in $\{1, \ldots, N\}$. Setting

$$
-\Delta u^{*}=-\Delta g \text { in } \cup_{j=1}^{N} O_{j}
$$

according to Remark 5, we see that the solution of the resulting problem satisfies interior Poisson-Dirichlet problems in the objects coupled with an exterior Poisson-Dirichlet problem by the transmission conditions (28). As a consequence, 
it exists and is uniquely defined in the space $H$. Finally, the system of equations satisfied by the initial approximation $u^{(0)}$,

$$
\left\{\begin{array}{c}
-\Delta u^{(0)}=f \text { in } \Omega, \\
u^{(0)}=0 \text { on } \partial \Omega,
\end{array}\right.
$$

defines a problem admitting a unique weak solution in $H$.

In addition, one may note that solutions to problem (21)-(22) satisfying (28) vanish in $\cup_{j=1}^{N} \overline{O_{j}}$. This observation allows to characterise the Hilbert space framework associated with the method of reflections applied to the PoissonDirichlet problem.

Proposition 4.3 Let $H=H_{0}^{1}(\Omega)$, equipped with the inner product

$$
a(u, v)=\int_{\Omega} \nabla u(x) \cdot \nabla v(x) \mathrm{d} x .
$$

For the Laplace operator completed with Dirichlet boundary conditions, the projectors $P_{M}$ and $P_{V}$ (resp. $P_{M_{i}}$ and $P_{V_{i}}$, with $i$ in $\{1, \ldots, N\}$ ) introduced in Subsection 3.2 are well-defined and orthogonal with respect to $a$, and their respective ranges are characterised as

$$
M=\left\{w \in H \mid w=0 \text { in } \cup_{j=1}^{N} \overline{O_{j}}\right\} \text { and } V=\left\{v \in H \mid \Delta v=0 \text { in } \Omega \backslash \cup_{j=1}^{N} \overline{O_{j}}\right\}
$$

(resp. $M_{i}=\left\{w \in H \mid w=0\right.$ in $\left.\overline{O_{i}}\right\}$ and $V_{i}=\left\{v \in H \mid \Delta v=0\right.$ in $\left.\left.\Omega \backslash \overline{O_{i}}\right\}, i=1, \ldots, N\right)$.

In addition, one has $V=\sum_{j=1}^{N} V_{j}$.

Proof. Let us first deal with the projector $P_{M}$. For any $u$ in $H, w=P_{M}(u)$ is by definition the weak solution in $H$ to the following exterior-interior transmission problem

$$
\left\{\begin{array}{l}
-\Delta w=-\Delta u \text { in } \Omega \backslash \cup_{j=1}^{N} \overline{O_{j}}, \\
w=0 \text { on } \partial \Omega, \\
w=0 \text { on } \partial O_{j}, j=1, \ldots, N, \\
{[w]=0 \text { across } \partial O_{j}, j=1, \ldots, N,} \\
-\Delta w=0 \text { in } \cup_{j=1}^{N} O_{j} .
\end{array}\right.
$$

We observe that the restriction of $w$, solution to (29), to the exterior of the objects satisfies a problem for a Poisson equation with homogeneous Dirichlet boundary conditions, and that its restriction to the interior of a given object solves a problem for the Laplace equation with a homogeneous Dirichlet boundary condition. The above problem thus makes sense for $u$ and $w$ in $H$, and is well-posed by virtue of the Lax-Milgram lemma. The well-posedness of the problem then implies that functions in the range of the projector vanish in the interiors of the objects, from which the characterisation of the subspace $M$ follows.

Using these facts and the weak formulation associated with the problem, one has

$$
\begin{aligned}
\forall u \in H, \forall w \in M, a\left(P_{M} u, w\right) & =\int_{\Omega \backslash \cup_{j=1}^{N} \overline{O_{j}}} \nabla P_{M} u(x) \cdot \nabla w(x) \mathrm{d} x \\
& =\left\langle-\Delta P_{M} u, w\right\rangle_{H^{-1}\left(\Omega \backslash \cup_{j=1}^{N} \overline{O_{j}}\right), H_{0}^{1}\left(\Omega \backslash \cup_{j=1}^{N} \overline{O_{j}}\right)} \\
& =\langle-\Delta u, w\rangle_{H^{-1}\left(\Omega \backslash \cup_{j=1}^{N} \overline{\bar{j}_{j}}\right), H_{0}^{1}\left(\Omega \backslash \cup_{j=1}^{N} \overline{\bar{O}_{j}}\right)} \\
& =\int_{\Omega \backslash \cup_{j=1}^{N} \overline{O_{j}}} \nabla u(x) \cdot \nabla w(x) \mathrm{d} x \\
& =a(u, w),
\end{aligned}
$$

so that $P_{M}$ and $P_{V}=I d-P_{M}$ are orthogonal projectors. 
Consider next the subset $V$ of $H$, orthogonal complement to $M$. One has that $P_{V}=I d_{H}-P_{M}$, so that, for any $u$ in $H$, the function $v=P_{V} u$ is a weak solution to the following system

$$
\left\{\begin{array}{l}
-\Delta v=0 \text { in } \Omega \backslash \cup_{j=1}^{N} \overline{O_{j}}, \\
v=0 \text { on } \partial \Omega, \\
v=u \text { on } \partial O_{j}, j=1, \ldots, N, \\
{[v]=0 \text { across } \partial O_{j}, j=1, \ldots, N,} \\
-\Delta v=-\Delta u \text { in } \cup_{j=1}^{N} O_{j},
\end{array}\right.
$$

yielding the characterisation of $V$.

The orthogonality of the projectors $P_{M_{i}}$ and $P_{V_{i}}$, and the characterizations of the respective associated subspaces $M_{i}$ and $V_{i}$, with $i$ in $\{1, \ldots, N\}$, are obtained in the same way.

Let us finally show that $V=\sum_{j=1}^{N} V_{j}$. Any element $v$ of $V$ may be represented by the sum of double layer potentials and Newton potentials, that is

$$
\text { a. e. } x \in \Omega \backslash \cup_{j=1}^{N} \partial O_{j}, v(x)=\sum_{j=1}^{N} \int_{\partial O_{j}} G(x, y)\left[\frac{\partial v}{\partial \mathbf{n}}\right](y) \mathrm{d} S(y)-\sum_{j=1}^{N} \int_{O_{j}} G(x, y) \Delta v(y) \mathrm{d} y,
$$

where the kernel $G$ is the Green function ${ }^{14}$ of the Laplace equation for the region $\Omega$, and the various integrals are understood in the sense of duality products. This provides the decomposition $v=\sum_{j=1}^{N} v_{j}$ by setting

$$
\forall i \in\{1, \ldots, N\}, v_{i}(x)=\int_{\partial O_{i}} G(x, y)\left[\frac{\partial v}{\partial \mathbf{n}}\right](y) \mathrm{d} S(y)-\int_{O_{j}} G(x, y) \Delta v(y) \mathrm{d} y .
$$

For each integer $i$ in $\{1, \ldots, N\}$, it is then easily seen that $v_{i}$ belongs to $V_{i}$, concluding the proof.

The assumptions of Theorems 4.1 and 4.2 being satisfied in view of the above result, both the sequential and averaged parallel form of the method converge to the solution.

Remark 6 In the present case, the orthogonality between the subspaces $V$ and $M$ (resp. $V_{i}$ and $M_{i}$ ) may be related to the orthogonality that exists between gradients of functions that are harmonic in a regular bounded open set $\Omega$ in $\mathbb{R}^{d}$ and gradients of functions in $H_{0}^{1}(\Omega)$, with respect to the $L^{2}\left(\Omega, \mathbb{R}^{d}\right)$-inner product.

Laplace problem with boundary conditions of the fourth type. By this name, we refer to the nonlocal boundary conditions used in boundary value problem (1)-(2)-(3)-(4) considered in Subsection 2.1, which correspond to homogeneous conditions for the boundary operators

$$
\mathscr{B}_{j}(u)=u_{\left.\right|_{\partial o_{j}}}-f_{\partial O_{j}} u(x) \mathrm{d} S(x), j=1, \ldots, N,
$$

where $f_{\partial O_{j}} u(x) \mathrm{d} S(x)$ denotes the mean of the function $u$ on the hypersurface $\partial O_{j}$.

As for the preceding Poisson-Dirichlet problem, we extend the boundary value problem to the interior of the objects by continuity, imposing that

$$
\left[u^{*}\right]=0 \text { across } \partial O_{j}, j=1, \ldots, N .
$$

We may then look for a solution $u^{*}$ in $H=H_{0}^{1}(\Omega)$. The boundary conditions on the objects being homogeneous, a suitable lifting is any function in $H$ having constant value on the boundaries of the objects, for instance $g \equiv 0$. Finally, as the given conditions on the boundaries of the objects correspond to a source term with support on these boundaries (see [Luk89, HV18]), we consequently require the initialisation $u^{(0)}$ to be a function in $H$ satisfying

$$
-\Delta u^{(0)}=\sum_{j=1}^{N} Q_{j} \frac{\mathscr{H}_{l_{\partial o_{j}}^{d-1}}}{\mathscr{H}^{d-1}\left(\partial O_{j}\right)} \text { in } \Omega
$$

\footnotetext{
${ }^{14}$ We recall that the Green function $G$ is such that

$$
\forall(x, y) \in \Omega \times \Omega, x \neq y, G(x, y)=\Phi(y-x)-\varphi_{x}(y),
$$
}

the function $\Phi$ being the fundamental solution of the Laplace equation and $\varphi_{x}$ being a corrector function which, for a fixed $x$ in $\Omega$, satisfies $\Delta \varphi_{x}=0$ in $\Omega$ and $\varphi_{x}=\Phi(\cdot-x)$ on $\partial \Omega$. 
Note that such a function exists, as one can be constructed by summing solutions to one-object problems.

We have the following result.

Proposition 4.4 Let $H=H_{0}^{1}(\Omega)$, equipped with the inner product

$$
a(u, v)=\int_{\Omega} \nabla u(x) \cdot \nabla v(x) \mathrm{d} x .
$$

For the Laplace operator completed with fourth-type boundary conditions, the projectors $P_{M}$ and $P_{V}$ (resp. $P_{M_{i}}$ and $P_{V_{i}}$, with $i$ in $\{1, \ldots, N\})$ introduced in Subsection 3.2 are well-defined and orthogonal with respect to $a$, and their respective ranges are characterised as

$$
M=\left\{w \in H \mid \forall j \in\{1, \ldots, N\}, \exists c_{j} \in \mathbb{R}, w=c_{j} \text { in } \overline{O_{j}}\right\}
$$

and

$$
V=\left\{v \in H \mid \Delta v=0 \text { in } \Omega \backslash \cup_{j=1}^{N} \overline{O_{j}}, \forall j \in\{1, \ldots, N\}, \int_{\partial O_{j}} \frac{\partial v}{\partial n}(x) \mathrm{d} S(x)=0\right\} .
$$

(resp. $M_{i}=\left\{w \in H \mid \exists c_{i} \in \mathbb{R}, w=c_{i}\right.$ in $\left.\overline{O_{i}}\right\}$

$$
\text { and } \left.V_{i}=\left\{v \in H \mid \Delta v=0 \text { in } \Omega \backslash \overline{O_{i}}, \int_{\partial O_{i}} \frac{\partial v}{\partial n}(x) \mathrm{d} S(x)=0\right\}, i=1, \ldots, N\right) \text {. }
$$

In addition, one has $V=\sum_{j=1}^{N} V_{j}$.

Proof. We proceed as in the proof of the preceding proposition. For any $u$ in $H$, the function $w=P_{M} u$ is the weak solution to the following system

$$
\left\{\begin{array}{l}
-\Delta w=-\Delta u \text { in } \Omega \backslash \cup_{j=1}^{N} \overline{O_{j}}, \\
w=0 \text { on } \partial \Omega, \\
\int_{\partial O_{j}} \frac{\partial w}{\partial n}(x) \mathrm{d} S(x)=\int_{\partial O_{j}} \frac{\partial u}{\partial n}(x) \mathrm{d} S(x), j=1, \ldots, N, \\
w_{\mid \partial O_{j}}-f_{\partial O_{j}} w(x) \mathrm{d} S(x)=0, \text { on } \partial O_{j}, j=1, \ldots, N, \\
{[w]=0 \text { across } \partial O_{j}, j=1, \ldots, N,} \\
-\Delta w=0 \text { in } \cup_{j=1}^{N} O_{j} .
\end{array}\right.
$$

Existence and uniqueness of a solution to the exterior problem are a well-known fact (see [Li89] for instance). The well-posedness of the interior Laplace-Dirichlet problem satisfied by the solution then implies that it is constant in each of the objects, a fact from which the characterisation of the space $M$ follows.

Let us now prove that that $P_{M}$ is an orthogonal projector. One has, integrating by parts and using the problem defining the projector,

$$
\begin{aligned}
\forall u \in H, \forall w \in M, a\left(P_{M} u, w\right) & =\int_{\Omega \backslash \cup_{j=1}^{N} \overline{O_{j}}} \nabla P_{M} u(x) \cdot \nabla w(x) \mathrm{d} x \\
& =\left\langle-\Delta P_{M} u, w\right\rangle_{H^{-1}\left(\Omega \backslash \cup_{j=1}^{N} \overline{O_{j}}\right), H_{0}^{1}\left(\Omega \backslash \cup_{j=1}^{N} \overline{O_{j}}\right)}+\sum_{j=1}^{N}\left\langle\frac{\partial P_{M} u}{\partial n}, w\right\rangle_{H^{-1 / 2}\left(\partial O_{j}\right), H^{1 / 2}\left(\partial O_{j}\right)} \\
& =\langle-\Delta u, w\rangle_{H^{-1}\left(\Omega \backslash \cup_{j=1}^{N} \overline{O_{j}}\right), H_{0}^{1}\left(\Omega \backslash \cup_{j=1}^{N} \overline{O_{j}}\right)}+\sum_{j=1}^{N} w_{{\overline{O_{j}}}_{j}} \int_{\partial O_{j}} \frac{\partial u}{\partial n}(x) \mathrm{d} S(x) \\
& =\int_{\Omega \backslash \cup_{j=1}^{N} \overline{O_{j}}} \nabla u(x) \cdot \nabla w(x) \mathrm{d} x \\
& =a(u, w),
\end{aligned}
$$

since the $w$ is constant on the boundaries of the objects. 
To characterise the subspace $V$, let $v$ be an element of the set on the right hand side of the identity (30). For any function $w$ in $M$, one has

$$
a(v, w)=\langle-\Delta v, w\rangle_{H^{-1}\left(\Omega \backslash \cup_{j=1}^{N} \overline{O_{j}}\right), H_{0}^{1}\left(\Omega \backslash \cup_{j=1}^{N} \overline{o_{j}}\right)}+\sum_{j=1}^{N} w_{\left.\right|_{\overline{o_{j}}}} \int_{\partial O_{j}} \frac{\partial v}{\partial n}(x) \mathrm{d} S(x)=0
$$

Conversely, let $v$ be an element of the orthogonal complement of $M$. Then, for any function $w$ in $M$, one has

$$
0=a(v, w)=\langle-\Delta v, w\rangle_{H^{-1}\left(\Omega \backslash \cup_{j=1}^{N} \overline{O_{j}}\right), H_{0}^{1}\left(\Omega \backslash \cup_{j=1}^{N} \overline{O_{j}}\right)}+\sum_{j=1}^{N} w_{{\overline{o_{j}}}_{j}} \int_{\partial O_{j}} \frac{\partial v}{\partial n}(x) \mathrm{d}(x) S .
$$

Choosing a function $w$ in $M$ which vanishes in the objects, one obtains that $-\Delta v=0$ in $\Omega \backslash \cup_{j=1}^{N} \overline{O_{j}}$ in a weak sense. One then concludes by using a function $w$ which vanishes in all the objects except the $j$ th one and by varying the integer $j$ to obtain the remaining conditions.

Here again, the orthogonality of the projectors $P_{M_{i}}$, and the characterizations of the subspaces $M_{i}$ and their orthogonal complements $V_{i}$, with $i$ in $\{1, \ldots, N\}$, are obtained similarly.

It remains to prove $V=\sum_{j=1}^{N} V_{j}$. One has

$$
\forall v \in V \text {, a. e. } x \in \Omega \backslash \cup_{j=1}^{N} \partial O_{j}, v(x)=\sum_{j=1}^{N} \int_{\partial O_{j}} G(x, y)\left[\frac{\partial v}{\partial \boldsymbol{n}}\right](y) \mathrm{d} S(y)-\sum_{j=1}^{N} \int_{O_{j}} G(x, y) \Delta v(y) \mathrm{d} y,
$$

with

$$
\forall j \in\{1, \ldots, N\}, \int_{\partial O_{j}} \frac{\partial v}{\partial \boldsymbol{n}}(x) \mathrm{d} S(x)=0
$$

Setting

$$
\forall i \in\{1, \ldots, N\}, v_{i}(x)=\int_{\partial O_{i}} G(x, y)\left[\frac{\partial v}{\partial \boldsymbol{n}}\right](y) \mathrm{d} S(y)-\int_{O_{i}} G(x, y) \Delta v(y) \mathrm{d} y
$$

then provides the adequate decomposition.

It follows from this result that Theorems 4.1 and 4.2 apply, so that the sequential and averaged parallel versions converge.

Remark 7 Continuing with the electrostatics analogy started in Subsection 2.1, a function $v$ in the subspace $V_{i}$ is usually said to be the potential an electric dipole, since the quantity

$$
\int_{\partial O_{i}} \frac{\partial v}{\partial n}(x) \mathrm{d} S(x)
$$

which corresponds to the total charge of the ith object, vanishes.

\subsubsection{The mobility problem for the Stokes equations}

In [Luk89], Luke analysed the sequential form of the method of reflections and proved its convergence when applied to the solution of a system of equations modelling the motion of a sedimenting suspension in a container, the so-called mobility problem for the Stokes equations. It is similar to, but more complex than, the Laplace problem with boundary condition of the fourth type we previously dealt with. We show how this problem fits into the orthogonal projection framework previously introduced. Proofs of the statement are omitted as they can be found in [Luk89] or adapted from [Höf18, NS20]. Note that a similar analysis for the Stokes equations with Dirichlet boundary conditions and a particle configuration set in the whole space can be found in [HV18].

Denoting by $\Omega$ the container, that is, a bounded, connected, open set of $\mathbb{R}^{3}$ with a smooth boundary, and by $O_{i} \subset \Omega$, with $i$ in $\{1, \ldots, N\}$, the rigid particles of arbitrary shape, which are connected open sets with smooth boundaries, such that their closures are non overlapping, the set $\cup_{j=1}^{N} O_{j}$ is called the solid phase of the suspension, while the set $\Omega \backslash \overline{\cup_{j=1}^{N} O_{j}}$ is called the fluid phase.

The problem is extended flow inside the particles by requiring the flow field to be continuous across the particle boundaries and the Stokes equations to also hold inside the particles, the inertialess motion of the rigid particles due to 
externally imposed forces and torques is then described by the fluid velocity field $\boldsymbol{u}$ and the pressure field $p$ satisfying the interior-exterior transmission problem

$$
\begin{aligned}
& -\mu \Delta u+\nabla p=0 \text { in } \Omega \backslash \cup_{j=1}^{N} \overline{O_{j}}, \\
& \operatorname{div} u=0 \text { in } \Omega \backslash \cup_{j=1}^{N} \overline{O_{j}}, \\
& u=0 \text { on } \partial \Omega, \\
& \forall x \in \partial O_{j}, u(x)=U_{O_{j}}+\omega_{O_{j}} \times\left(x-x_{O_{j}}\right), j=1, \ldots, N, \\
& \int_{\partial O_{j}}[\boldsymbol{\sigma}(u)] \cdot \boldsymbol{n}(y) \mathrm{d} S(y)=F_{O_{j}}, j=1, \ldots, N, \\
& \int_{\partial O_{j}}\left(y-x_{O_{j}}\right) \times[\boldsymbol{\sigma}(u)] \cdot \boldsymbol{n}(y) \mathrm{d} S(y)=\tau_{O_{j}}, j=1, \ldots, N, \\
& {[u]=0 \text { across } \partial O_{j}, j=1, \ldots, N,} \\
& -\mu \Delta u+\nabla p=0 \text { in } \cup_{j=1}^{N} O_{j}, \\
& \operatorname{div} u=0 \text { in } \cup_{j=1}^{N} O_{j},
\end{aligned}
$$

where $\mu$ is the kinematic viscosity coefficient (which will be set to 1 in what follows for simplicity), $\sigma(u)$ is the stress tensor, $\boldsymbol{\sigma}(u)=\mu\left(\nabla u+(\nabla u)^{T}\right)-p \mathbf{I}, \boldsymbol{n}$ is the outward pointing unit normal vector to the particle boundaries, and the instantaneous linear and angular velocities $U_{O_{i}}$ and $\omega_{O_{i}}$ of the $i$ th particle are unknowns in $\mathbb{R}^{3}$ to be determined (along with the fluid velocity and pressure), whereas the centres of mass $x_{O_{i}}$ of the particles, the total forces $F_{O_{i}}$ and the total torques $\tau_{O_{i}}$, with $i$ in $\{1, \ldots, N\}$, applied to the particles are given ${ }^{15}$. In the above system, the trivial flow is used as an admissible lifting of the homogeneous boundary data.

The variational formulation of the above problem allows to reduce it to that of finding solely the velocity field $u$ in the space

$$
H=H_{0, \sigma}^{1}(\Omega):=\left\{v \in H_{0}^{1}\left(\Omega, \mathbb{R}^{3}\right) \mid \operatorname{div} v=0 \text { in } \mathscr{D}^{\prime}(\Omega)\right\},
$$

as the pressure $p$ can recovered (up to a constant) from it, which is equipped with the bilinear, symmetric, continuous and coercive form

$$
\forall(u, v) \in H \times H, a(u, v)=\int_{\Omega} \nabla u(x): \nabla v(x) \mathrm{d} x .
$$

As an initialisation, one chooses a field $u^{(0)}$ satisfying the equations of the above system except for the boundary conditions on the surface of the particles, meaning that associated the fluid flow will satisfy the constraints of the forces acting on the particles but will fail to have a rigid motion in the particles.

In such a setting, the elements of the subspace $M$ are the elements of $H$ which achieve a rigid motion in the particles, i.e.,

$$
M=\left\{w \in H \mid \exists U \in\left(\mathbb{R}^{3}\right)^{N}, \exists \omega \in\left(\mathbb{R}^{3}\right)^{N}, \forall j \in\{1, \ldots, N\}, \forall x \in \overline{O_{j}}, w(x)=U_{j}+\omega_{j} \times\left(x-x_{O_{j}}\right)\right\},
$$

the orthogonal complement of $M$ being

$$
\begin{aligned}
& V=\left\{v \in H \mid \exists p \in L^{2}(\Omega), \Delta v+\nabla p=0 \text { in } \Omega \backslash \cup_{j=1}^{N} \overline{O_{j}},\right. \\
& \left.\qquad \forall j \in\{1, \ldots, N\}, \int_{\partial O_{j}}[\boldsymbol{\sigma}(v)] \cdot \boldsymbol{n}(y) \mathrm{d} S(y)=0 \text { and } \int_{\partial O_{j}}\left(y-x_{O_{j}}\right) \times[\boldsymbol{\sigma}(v)] \cdot \boldsymbol{n}(y) \mathrm{d} S(y)=0\right\} .
\end{aligned}
$$

It then follows that, for any integer $i$ in $\{1, \ldots, N\}$,

$$
\forall i \in\{1, \ldots, N\}, M_{i}=\left\{w \in H \mid \exists U \in \mathbb{R}^{3}, \exists \omega \in \mathbb{R}^{3}, \forall x \in \overline{O_{i}}, w(x)=U_{i}+\omega_{i} \times\left(x-x_{O_{i}}\right)\right\},
$$

and

$$
\begin{aligned}
& \forall i \in\{1, \ldots, N\}, V_{i}=\left\{v \in H \mid \exists p \in L^{2}(\Omega), \Delta v+\nabla p=0 \text { in } \Omega \backslash \overline{O_{i}},\right. \\
& \left.\qquad \int_{\partial O_{i}}[\boldsymbol{\sigma}(v)] \cdot \boldsymbol{n}(y) \mathrm{d} S(y)=0 \text { and } \int_{\partial O_{i}}\left(y-x_{O_{i}}\right) \times[\boldsymbol{\sigma}(v)] \cdot \boldsymbol{n}(y) \mathrm{d} S(y)=0\right\},
\end{aligned}
$$

the elements of the subspaces $V_{i}$ being the hydrodynamic analogues of the electric dipoles. The fact that the $\operatorname{sum} \sum_{j=1}^{N} V_{j}$ is closed is established by Luke.

\footnotetext{
${ }^{15}$ For instance, for suspensions sedimenting in a uniform gravitational field, one has $F_{O_{i}}=m_{O_{i}} G$, where $m_{O_{i}}$ is the mass of the particle adjusted for buoyancy and $G$ is the gravitational acceleration, and $\tau_{O_{i}}=0$.
} 
Remark 8 When $\Omega=\mathbb{R}^{3}$ and the particles are identical spheres of radius $R$, one can characterise in a more precise way both the projections onto $M_{i}$ and the functions in $V_{i}$, with $i$ in $\{1, \ldots, N\}$, using closed-form expressions, like Stokes' law, to explicitly compute the drag force exerted on a particle (see [Höf18, NS20]). In such a case, $H$ is the subspace $\boldsymbol{H}_{\sigma}^{1}$ of all divergence free functions in the homogeneous Sobolev space $\dot{H}^{1}\left(\mathbb{R}^{3}, \mathbb{R}^{3}\right.$ ) (defined as the closure of $\mathscr{C}_{c}^{\infty}\left(\mathbb{R}^{3}, \mathbb{R}^{3}\right.$ ) with respect to the $L^{2}$-norm of the gradient) and, for a given function $u$ in $H$, the projection of $u$ onto $M_{i}$ satisfies $P_{M_{i}} u(x)=U+\omega_{O_{i}} \times\left(x-x_{O_{i}}\right)$ for any $x$ in $\overline{O_{i}}$, with

$$
U=\frac{1}{4 \pi R^{2}} \int_{\partial O_{i}} u(x) \mathrm{d} S(x) \text { and } \omega_{O_{i}}=\frac{3}{8 \pi R^{4}} \int_{\partial O_{i}}\left(x-x_{O_{i}}\right) \times u(x) \mathrm{d} S(x) .
$$

Moreover, for any function $v$ in $V_{i}$, one has

$$
\int_{\partial O_{i}} v(x) \mathrm{d} S(x)=0 \text { and } \int_{\partial O_{i}}\left(x-x_{O_{i}}\right) \times v(x) \mathrm{d} S(x)=0 .
$$

\subsection{An example of non-orthogonal case}

We now consider a problem for the Laplace equation set in an unbounded domain, each object having either a Dirichlet or a Neumann condition imposed on its boundary. For such a configuration, we were unable to prove the orthogonality of the projection operators, and cases of divergence for the sequential form of the method are actually observed in numerical tests in dimension two (see Subsection 5.2). This leads us to conjecture that the convergence theory proposed in the present work does not apply in this case, which is thus called "non-orthogonal".

Nevertheless, assuming as in Proposition 3.2 that $H$ is a Banach space, one can find sufficient conditions for the convergence of the sequential version of the method. To do this, we follow the approach used by Balabane in [Bal04] for the parallel form of the method of reflections, and prove that, under a certain geometrical condition, the series defining the approximation converges to the solution to the boundary value problem under consideration.

In the present subsection, this domain $\Omega$ is equal to $\mathbb{R}^{3}$ and the $N$ objects are disjoints compact sets in $\Omega$, with boundaries of class $\mathscr{C}^{2}$. Given two positive integers $N_{D}$ and $N_{N}$ such that $N=N_{D}+N_{N}$, the objects associated with a Dirichlet boundary condition are numbered from 1 to $N_{D}$ and that the ones associated with a Neumann boundary condition are numbered form $N_{D}+1$ to $N$. Then, given $N_{D}$ functions $b_{i}^{D}, i=1, \ldots, N_{D}$, and $N_{N}$ functions $b_{i}^{N}, i=1, \ldots, N_{N}$, we look for a function $u$ satisfying

$$
\begin{aligned}
& -\Delta u=0 \text { in } \mathbb{R}^{3} \backslash \cup_{j=1}^{N} \overline{O_{j}}, \\
& u=b_{j}^{D} \text { on } \partial O_{j}, j=1, \ldots, N_{D}, \\
& \frac{\partial u}{\partial \boldsymbol{n}}=b_{j}^{N} \text { on } \partial O_{N_{D}+j}, j=1, \ldots, N_{N} .
\end{aligned}
$$

Observe that this boundary value problem is an exterior one (it is set in the complement of a union of bounded sets), and necessitates the introduction of a weighted Sobolev space to properly define its solution (see [AGG97] or [DL00, XI, B]). For any subset $\omega$ of $\Omega$, we set

$$
W(\omega)=\left\{v: \omega \rightarrow \mathbb{R} \mid \int_{\Omega} \frac{v^{2}(x)}{1+\|x\|^{2}} \mathrm{~d} x<+\infty, \nabla v \in L^{2}\left(\omega, \mathbb{R}^{3}\right)\right\} .
$$

A first step in proving the convergence of the method is to establish some boundary estimates for the relections.

Lemma 4.5 Consider the sequence of reflections $\left(u_{i}^{(k)}\right)_{k \in \mathbb{N}, i \in\{1, \ldots, N\}}$ generated by the sequential form of the method of reflections applied to the solution of problem (31) and define the associated sequence of scalars

$$
\forall k \in \mathbb{N}^{*}, \forall(i, j) \in\{1, \ldots, N\}^{2}, a_{i, j}^{(k)}=\left\|u_{i}^{(k)}\right\|_{H^{1}\left(\partial O_{j}\right)}^{2}+\left\|\frac{\partial u_{i}^{(k)}}{\partial \boldsymbol{n}}\right\|_{L^{2}\left(\partial O_{j}\right)}^{2} .
$$

Then, for any pair of distinct integers $i$ and $j$ in $\{1, \ldots, N\}$, there exists a positive constant $\kappa_{i, j}$ depending on the geometry of the problem such that

$$
\forall k \in \mathbb{N}^{*}, a_{i, j}^{(k+1)} \leq N \kappa_{i, j}\left(\sum_{m=1}^{i-1} a_{m, i}^{(k+1)}+\sum_{m=i+1}^{N} a_{m, i}^{(k)}\right) .
$$


Proof. First, we remark that, for any integer $k$ in $\mathbb{N}^{*}$, the datum of the boundary value problem defining a reflection is the trace (for a reflection associated to an object with a Dirichlet boundary condition) or the trace of the normal derivative (for a reflection associated to an object with a Neumann condition) on an interior curve of a sum of harmonic functions. It follows from Weyl's lemma on the interior regularity of harmonic functions and from results in Chapter 2 of [Gri85] that these reflections enjoy smoothness properties which, using a trace continuity theorem (see Chapter 1 of [Gri85] for instance), allow to show there exist positive constants $C_{i}$ depending only on the geometry such that

$$
\forall k \in \mathbb{N}^{*}, \forall i \in\left\{1, \ldots, N_{D}\right\},\left\|\frac{\partial u_{i}^{(k)}}{\partial \boldsymbol{n}}\right\|_{L^{2}\left(\partial O_{i}\right)} \leq C_{i}\left\|u_{i}^{(k)}\right\|_{H^{1}\left(\partial O_{i}\right)},
$$

and

$$
\forall k \in \mathbb{N}^{*}, \forall i \in\left\{N_{D}+1, \ldots, N\right\},\left\|u_{i}^{(k)}\right\|_{H^{1}\left(\partial O_{i}\right)} \leq C_{i}\left\|\frac{\partial u_{i}^{(k)}}{\partial \boldsymbol{n}}\right\|_{L^{2}\left(\partial O_{i}\right)} .
$$

From now on, we shall assume, without loss of generality, that $C_{i} \geq 1$, with $i$ in $\{1, \ldots, N\}$.

Next, for any integers $k$ in $\mathbb{N}^{*}$ and $i$ in $\{1, \ldots, N\}$, the reflection $u_{i}^{(k)}$ is extended to the whole of $\Omega$ by requiring its extension $\tilde{u}_{i}^{(k)}$ to belong to the weighted Sobolev space $W(\Omega)$, to satisfy the same equations as $u_{i}^{(k)}$ in the complement of $O_{i}$ and the Laplace equation in $O_{i}$, and to have a vanishing jump across $\partial O_{i}$ if $1 \leq i \leq N_{D}$, or a vanishing jump of its normal derivative across $\partial O_{i}$ if $N_{D}+1 \leq i \leq N$. Note that the interior problem associated with a reflection satisfying a Neumann boundary condition is indeed well-posed, since its normal derivative has a zero mean value on $\partial O_{i}$. We may then define the space

$$
\begin{aligned}
H=H=\{v \in W(\Omega) \mid- & \Delta v=0 \text { in } \mathbb{R}^{3} \backslash \cup_{j=1}^{N} \partial O_{j},[v]=0 \text { on } \partial O_{j}, j=1, \ldots, N_{D}, \\
& {\left.\left[\frac{\partial v}{\partial \boldsymbol{n}}\right]=0 \text { on } \partial O_{j}, \int_{\partial O_{j}} \frac{\partial v}{\partial \boldsymbol{n}}(y) \mathrm{d} S(y)=0 \text { and } \int_{\partial O_{j}} v_{\mathrm{lo}_{j}}(y) \mathrm{d} S(y)=0, j=N_{D}+1, \ldots, N\right\}, }
\end{aligned}
$$

and its subspaces

$$
\forall i \in\{1, \ldots, N\}, V_{i}=\left\{w \in H \mid-\Delta w=0 \text { in } \Omega \backslash \partial O_{i}\right\} .
$$

Functions $v$ in $H$ have an explicit formulation in terms of their jumps on the boundaries $\partial O_{i}$, with $i$ in $\{1, \ldots, N\}$, due to the following integral representation

$$
\forall x \in \Omega \backslash \cup_{j=1}^{N} \partial O_{j}, v(x)=\frac{1}{4 \pi} \sum_{j=N_{D}+1}^{N} \int_{\partial O_{j}} \frac{(x-y) \cdot \boldsymbol{n}(y)}{\|x-y\|^{3}}[v(y)] \mathrm{d} S(y)-\frac{1}{4 \pi} \sum_{j=1}^{N_{D}} \int_{\partial O_{j}} \frac{1}{\|x-y\|}\left[\frac{\partial v}{\partial \boldsymbol{n}}(y)\right] \mathrm{d} S(y),
$$

and the same goes for their gradient,

$$
\begin{array}{r}
\forall x \in \Omega \backslash \cup_{j=1}^{N} \partial O_{j}, \nabla v(x)=\frac{1}{4 \pi} \sum_{j=N_{D}+1}^{N} \int_{\partial O_{j}} \frac{1}{\|x-y\|^{3}}\left(\boldsymbol{n}(y)-3 \frac{(x-y) \cdot \boldsymbol{n}(y)}{\|x-y\|^{2}}(x-y)\right)[v(y)] \mathrm{d} S(y) \\
+\frac{1}{4 \pi} \sum_{j=1}^{N_{D}} \int_{\partial O_{j}} \frac{x-y}{\|x-y\|^{3}}\left[\frac{\partial v}{\partial \boldsymbol{n}}(y)\right] \mathrm{d} S(y) .
\end{array}
$$

For any integer $i$ in $\{1, \ldots, N\}$, let us denote by $S_{i}$ the area of $\partial O_{i}$, that is $S_{i}=\mathscr{H}^{2}\left(\partial\left(O_{i}\right)\right.$, and set, for any pair of distinct integers $i$ and $j$ in $\{1, \ldots, N\}, d_{i, j}=\min _{(x, y) \in \partial O_{i} \times \partial O_{j}}\|x-y\|$. We will deal differently with the reflection according to the type of boundary condition it satisfies on the associated object.

For a reflection associated with an object with an imposed Dirichlet boundary condition, that is for an integer $i$ in $\left\{1, \ldots, N_{D}\right\}$, the jump condition across $\partial O_{i}$ yields

$$
\forall y \in \partial O_{i},\left[\frac{\partial \tilde{u}_{i}^{(k+1)}}{\partial \mathbf{n}}(y)\right]=\frac{\partial u_{i}^{(k+1)}}{\partial \mathbf{n}}(y)+\sum_{m=1}^{i-1} \frac{\partial u_{m}^{(k+1)}}{\partial \mathbf{n}}(y)+\sum_{m=i+1}^{N} \frac{\partial u_{m}^{(k)}}{\partial \boldsymbol{n}}(y) .
$$

On the other hand, since the function $\tilde{u}_{i}^{(k)}$ belongs to $V_{i}$, it follows from the integral representation formula (34) that

$$
\forall x \in \mathbb{R}^{3} \backslash \partial O_{i}, \tilde{u}_{i}^{(k+1)}(x)=-\frac{1}{4 \pi} \int_{\partial O_{i}} \frac{1}{\|x-y\|}\left[\frac{\partial \tilde{u}_{i}^{(k+1)}}{\partial \mathbf{n}}(y)\right]_{\partial O_{i}} \mathrm{~d} S(y)
$$


hence

$$
\begin{array}{r}
\forall x \in \mathbb{R}^{3} \backslash \partial O_{i}, \tilde{u}_{i}^{(k+1)}(x)=-\frac{1}{4 \pi} \int_{\partial O_{i}} \frac{1}{\|x-y\|} \frac{\partial u_{i}^{(k+1)}}{\partial \boldsymbol{n}}(y) \mathrm{d} S(y)-\frac{1}{4 \pi} \sum_{m=1}^{i-1} \int_{\partial O_{i}} \frac{1}{\|x-y\|} \frac{\partial u_{m}^{(k+1)}}{\partial \boldsymbol{n}}(y) \mathrm{d} S(y) \\
\quad-\frac{1}{4 \pi} \sum_{m=i+1}^{N} \int_{\partial O_{i}} \frac{1}{\|x-y\|} \frac{\partial u_{m}^{(k)}}{\partial \boldsymbol{n}}(y) \mathrm{d} S(y) .
\end{array}
$$

By means of the Cauchy-Schwarz inequality, one has, for any integer $j$ in $\{1, \ldots, N\}$ distinct from $i$,

$$
\forall x \in \partial O_{j},\left|u_{i}^{(k+1)}(x)\right| \leq \frac{\sqrt{S_{i}}}{4 \pi d_{i, j}}\left(\left\|\frac{\partial u_{i}^{(k+1)}}{\partial \boldsymbol{n}}\right\|_{L^{2}\left(\partial O_{i}\right)}+\sum_{m=1}^{i-1}\left\|\frac{\partial u_{m}^{(k+1)}}{\partial \mathbf{n}}\right\|_{L^{2}\left(\partial O_{i}\right)}+\sum_{m=i+1}^{N}\left\|\frac{\partial u_{m}^{(k)}}{\partial \boldsymbol{n}}\right\|_{L^{2}\left(\partial O_{i}\right)}\right),
$$

so that using (33), squaring both sides of the inequality and integrating over $\partial O_{j}$, we obtain

$$
\left\|u_{i}^{(k+1)}\right\|_{L^{2}\left(\partial O_{j}\right)}^{2} \leqslant \frac{N S_{i} S_{j} C_{i}^{2}}{(4 \pi)^{2} d_{i, j}^{2}}\left(\sum_{m=1}^{i-1} a_{m, i}^{(k+1)}+\sum_{m=i+1}^{N} a_{m, i}^{(k)}\right) .
$$

In addition, repeating these computations starting from (35), we get similar estimates the normal and tangential derivative traces,

$$
\begin{aligned}
& \left\|\frac{\partial u_{i}^{(k+1)}}{\partial \boldsymbol{n}}\right\|_{L^{2}\left(\partial O_{j}\right)}^{2} \leq \frac{N S_{i} S_{j} C_{i}^{2}}{(4 \pi)^{2} d_{i, j}^{4}}\left(\sum_{m=1}^{i-1} a_{m, i}^{(k+1)}+\sum_{m=i+1}^{N} a_{m, i}^{(k)}\right), \\
& \left\|\frac{\partial u_{i}^{(k+1)}}{\partial \tau}\right\|_{L^{2}\left(\partial O_{j}\right)}^{2} \leq \frac{N S_{i} S_{j} C_{i}^{2}}{(4 \pi)^{2} d_{i, j}^{4}}\left(\sum_{m=1}^{i-1} a_{m, i}^{(k+1)}+\sum_{m=i+1}^{N} a_{m, i}^{(k)}\right) .
\end{aligned}
$$

Likewise, for a reflection $\tilde{u}_{i}^{(k)}$ satisfying a Neumann boundary condition on $\partial O_{i}$, that is for any integer $i$ in $\left\{N_{D}+\right.$ $1, \ldots, N\}$, one has, one has

$$
\forall y \in \partial O_{i},\left[\tilde{u}_{i}^{(k+1)}(y)\right]=u_{i}^{(k+1)}(y)+\sum_{m=1}^{i-1} u_{m}^{(k+1)}(y)+\sum_{m=i+1}^{N} u_{m}^{(k)}(y)+\lambda_{i}^{(k+1)},
$$

where $\lambda_{i}^{(k+1)}$ is a real number chosen in such a way that the condition

$$
\int_{\partial O_{i}} \tilde{u}_{i}^{(k+1)}{ }_{o_{i}}(y) \mathrm{d} S(y)=0
$$

is satisfied. The integral representation formula then gives

$$
\begin{aligned}
\forall x \in \mathbb{R}^{3} \backslash \partial O_{i}, \tilde{u}_{i}^{(k+1)}(x)=\frac{1}{4 \pi} \int_{\partial O_{i}} & \frac{(x-y) \cdot \boldsymbol{n}(y)}{\|x-y\|^{3}} u_{i}^{(k+1)}(y) \mathrm{d} S(y)+\frac{1}{4 \pi} \sum_{m=1}^{i-1} \int_{\partial O_{i}} \frac{(x-y) \cdot \boldsymbol{n}(y)}{\|x-y\|^{3}} u_{m}^{(k+1)}(y) \mathrm{d} S(y) \\
& +\frac{1}{4 \pi} \sum_{m=i+1}^{N} \int_{\partial O_{i}} \frac{(x-y) \cdot \boldsymbol{n}(y)}{\|x-y\|^{3}} u_{m}^{(k)}(y) \mathrm{d} S(y)+\frac{\lambda_{i}^{(k+1)}}{4 \pi} \int_{\partial O_{i}} \frac{(x-y) \cdot \boldsymbol{n}(y)}{\|x-y\|^{3}} \mathrm{~d} S(y) .
\end{aligned}
$$

For any integer $j$ in $\{1, \ldots, N\}$ distinct from $i$, one then has

$$
\forall x \in \partial O_{j},\left|u_{i}^{(k+1)}(x)\right| \leq \frac{\sqrt{S_{i}}}{4 \pi d_{i, j}^{2}}\left(\left\|u_{i}^{(k+1)}\right\|_{L^{2}\left(\partial O_{i}\right)}+\sum_{m=1}^{i-1}\left\|u_{m}^{(k+1)}\right\|_{L^{2}\left(\partial O_{i}\right)}+\sum_{m=i+1}^{N}\left\|u_{m}^{(k)}\right\|_{L^{2}\left(\partial O_{i}\right)}\right),
$$

from which one gets

$$
\left\|u_{i}^{(k+1)}\right\|_{L^{2}\left(\partial O_{j}\right)}^{2} \leq \frac{N S_{i} S_{j} C_{i}^{2}}{(4 \pi)^{2} d_{i, j}^{4}}\left(\sum_{m=1}^{i-1} a_{m, i}^{(k+1)}+\sum_{m=i+1}^{N} a_{m, i}^{(k)}\right)
$$


In the same manner, one may obtain the following estimates

$$
\begin{aligned}
& \left\|\frac{\partial u_{i}^{(k+1)}}{\partial \boldsymbol{n}}\right\|_{L^{2}\left(\partial O_{j}\right)}^{2} \leq \frac{N S_{i} S_{j} C_{i}^{2}}{\pi^{2} d_{i, j}^{6}}\left(\sum_{m=1}^{i-1} a_{m, i}^{(k+1)}+\sum_{m=i+1}^{N} a_{m, i}^{(k)}\right), \\
& \left\|\frac{\partial u_{i}^{(k+1)}}{\partial \tau}\right\|_{L^{2}\left(\partial O_{j}\right)}^{2} \leq \frac{N S_{i} S_{j} C_{i}^{2}}{\pi^{2} d_{i, j}^{6}}\left(\sum_{m=1}^{i-1} a_{m, i}^{(k+1)}+\sum_{m=i+1}^{N} a_{m, i}^{(k)}\right) .
\end{aligned}
$$

Finally, summing estimates (36), (37) and (38) on the one hand, and estimates (39), (40) and (41) on the other hand, setting

$$
\kappa_{i, j}=\frac{S_{i} S_{j} C_{i}^{2}}{(4 \pi)^{2}} \min \left\{\frac{1}{d_{i, j}^{2}}+\frac{2}{d_{i, j}^{4}}, \frac{1}{d_{i, j}^{4}}+\frac{16}{d_{i, j}^{6}}\right\},
$$

and using the fact that the extension $\tilde{u}_{i}^{(k)}$ coincides with $u_{i}^{(k)}$ outside of $O_{i}$, we easily see that the claim holds whatever the type of condition imposed on the object boundary.

A convergence criterion can now be stated.

Theorem 4.6 Let $\kappa(N)=\max \left\{\kappa_{i, j} \mid(i, j) \in\{1, \ldots, N\}^{2}, i \neq j\right\}, \kappa_{i, j}$ being defined by (42), and assume that

$$
N(N-1) \kappa(N)<1 \text {. }
$$

Then, for any integer $i$ in $\{1, \ldots, N\}$, the series $\sum_{k=0}^{+\infty} u_{i}^{(k)}$, generated by the sequential form of the method of reflections applied to the solution of problem (31), converges in $W\left(\Omega \backslash \overline{O_{i}}\right)$, and its limits $u_{i}$ is such that the restriction of the sum $\sum_{i=1}^{N} u_{i}$ to $\Omega \backslash \cup_{j=1}^{N} \overline{O_{j}}$ is the unique solution to problem (31) in $W\left(\Omega \backslash \cup_{j=1}^{N} \overline{O_{j}}\right)$.

PRoof. Define the sequence $\left(\alpha_{\ell}\right)_{\ell \in \mathbb{N}^{*}}$ by

$$
\forall k \in \mathbb{N}^{*}, \forall i \in\{1, \ldots, N\}, \alpha_{i+(k-1) N}=\max _{j \in\{1, \ldots, N\} \backslash\{i\}} a_{i, j}^{(k)},
$$

the coefficients $a_{i, j}^{(k)}$ being defined in Lemma 4.5. Setting $\ell=i+k N$ for any integers $k$ in $\mathbb{N}^{*}$ and $i$ in $\{1, \ldots, N\}$, one has, owing to (32),

$$
\alpha_{\ell} \leq N(N-1) \kappa(N) \max _{m \in\{1, \ldots, N-1\}} \alpha_{\ell-m} \leq N(N-1) \kappa(N) \max _{m \in\{0, \ldots, N-1\}} \alpha_{\ell-m} .
$$

For $\ell>2 N$, denoting $m_{0}=\underset{m \in\{0, \ldots, N-1\}}{\arg \max } \alpha_{\ell-m}$, one then gets

$$
\alpha_{\ell-m_{0}} \leq N(N-1) \kappa(N) \max _{m \in\{0, \ldots, N-1\}} \alpha_{\ell-m_{0}-m}
$$

and, due to condition (43),

$$
N(N-1) \kappa(N) \max _{m \in\left\{0, \ldots, N-1-m_{0}\right\}} \alpha_{\ell-m_{0}-m}<\max _{m \in\left\{m_{0}, \ldots, N-1\right\}} \alpha_{\ell-m} \leq \alpha_{\ell-m_{0}} .
$$

This implies that

$$
\alpha_{\ell-m_{0}} \leq N(N-1) \kappa(N) \max _{m=N-m_{0}, \ldots, N-1} \alpha_{\ell-m_{0}-m} \leq N(N-1) \kappa(N) \max _{m=N, \cdots, N-1+m_{0}} \alpha_{\ell-m}
$$

so that finally

$$
\max _{m \in\{0, \ldots, N-1\}} \alpha_{\ell-m} \leq N(N-1) \kappa(N) \max _{m=0, \cdots, N-1} \alpha_{\ell-N-m} .
$$

As a consequence, for any integer $i$ in $\{1, \ldots, N\}$, the series $\sum_{k \in \mathbb{N}^{*}} u_{i}^{(k)}$ is convergent on the boundary of $\Omega \backslash \overline{O_{i}}$, and thus in $W\left(\Omega \backslash \cup_{j=1}^{N} \overline{O_{j}}\right)$. Moreover, one can check that its limit $u_{i}$ satisfies

$$
\left\{\begin{array}{l}
-\Delta u_{i}=0 \text { in } \Omega \backslash \cup_{j=1}^{N} \overline{O_{j}}, \\
u_{i}=U_{i}-\sum_{j=1}^{i-1} u_{j}-\sum_{j=i+1}^{N} u_{j} \text { on } \partial O_{i},
\end{array}\right.
$$


if the integer $i$ belongs to $\left\{1, \ldots, N_{D}\right\}$, or

$$
\left\{\begin{array}{l}
-\Delta u_{i}=0 \text { in } \Omega \backslash \cup_{j=1}^{N} \overline{O_{j}} \\
\frac{\partial u_{i}}{\partial \boldsymbol{n}}=W_{i-N_{D}}-\sum_{j=1}^{i-1} \frac{\partial u_{j}}{\partial \boldsymbol{n}}-\sum_{j=i+1}^{N} \frac{\partial u_{j}}{\partial \boldsymbol{n}} \text { on } \partial O_{i}
\end{array}\right.
$$

if $i$ belongs to $\left\{N_{D}+1, \ldots, N\right\}$. Summing the restrictions to $\Omega \backslash \cup_{j=1}^{N} \overline{O_{j}}$ of these limits, one concludes using linearity.

Remark 9 A similar analysis could be carried out when Robin boundary conditions are imposed on the objects, that is

$$
\mathscr{B}_{i}(u)=\frac{\partial u}{\partial \mathbf{n}}+\beta_{i} u, i=1, \ldots, N,
$$

where the coefficients $\beta_{i}$, with $i$ in $\{1, \ldots, N\}$, are real numbers, giving rise to sufficient convergence condition. Indeed, one can see that Neumann boundary conditions correspond to Robin conditions with $\beta_{i}=0$, while Dirichlet conditions amount to the limiting case of $\beta_{i}$ tending to $+\infty$. However, the question of the unconditional convergence of the sequential form (or of the averaged parallel form) of the method in the case of Robin boundary conditions using the same coefficient $\beta$ for each object is, as far as we know, open.

\section{Numerical experiments}

In this short section, we aim at confirming numerically the theoretical results obtained in the paper and investigating cases not handled by the previous analysis. More involved numerical tests of the method can be found in [CGH $\left.{ }^{+} 19\right]$.

The problems solved numerically by the method of reflections are for the Laplace equation in some interior and exterior domains of $\mathbb{R}^{2}$, with both Dirichlet and Neumann boundary conditions imposed. A publicly available MATLAB package ${ }^{16}$ was used for the numerical computations, which relied on an integral formulation of the problem solved by the Nyström method.

Note that, for problems set in bounded domains, similar results (which are not reprinted here) were obtained with a finite element code. However, employing this type of discretisation method with the method of reflections is not a sensible choice, as the computational effort required to solve any of the one-object problem is always higher than that of the many-object one, due to the fact that the mesh used for the problem is a subset of the meshes used for the subproblems (see Figure 1 for an illustration). This observation emphasizes the fact that, as a boundary decomposition method, the method of reflections is, in practice, better suited to discretisation methods based on boundary integral representation of the solution.
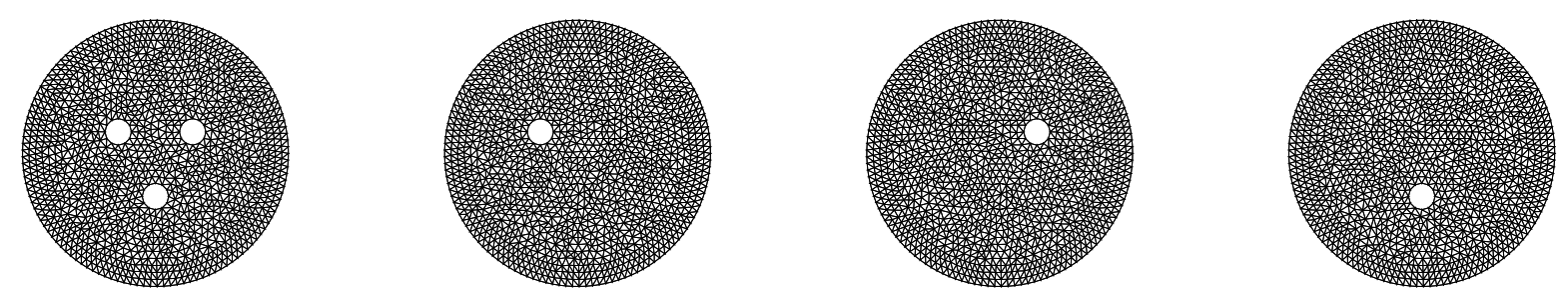

Figure 1: From left to right, finite element meshes for both the problem dealt with in Subsection 5.1 and its three associated one-object subproblems.

\subsection{Rate of convergence in a bounded domain}

The first numerical experiment concerns the rate of convergence of the method. It is inspired by a counterexample to the convergence of the parallel form of the method of reflections found in [IB01]. In two dimensions, we consider a bounded domain, namely a ball centred at the origin and with radius equal to 10, containing three objects, which are balls with identical radii equal to 1 and centres respectively set at the vertices of an equilateral triangle whose centroid lies at the origin. Using the length $l$ of a side of the triangle as a parameter, we investigate the convergence of the different forms of the method for solving a Dirichlet problem for the Laplace equation as $l$ varies.

\footnotetext{
${ }^{16}$ Integral Equation Solver (http://www.mathworks.com/matlabcentral/fileexchange/34241) by Alexandre Munnier and Bruno Pinçon, MATLAB Central File Exchange. Retrieved February 15, 2016.
} 
Figure 2 presents the relative error of the method as a function of the number of cycles for three distinct values of the parameter $l$. This relative error is based on the $\ell^{2}$-norm of the difference between the numerical solution for the full problem with that of the method of reflections after a given number of cycles computed at a finite number of points in the domain $\Omega \backslash \cup_{i=1}^{3} \overline{O_{i}}$. One can observe the sequential and the averaged parallel forms of the method are convergent in each case, as predicted by the theoretical results. The parallel form is seen to diverge for the smallest chosen value of $l$, but converges for larger values of the parameter.

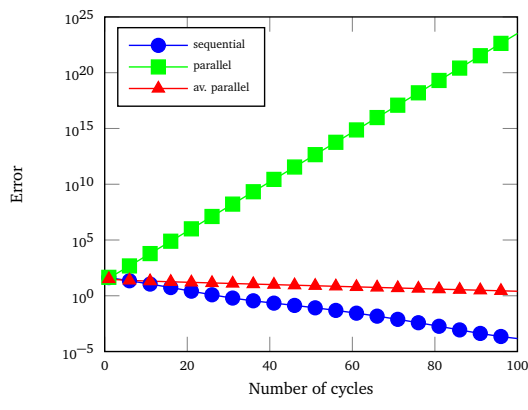

(a) $l=1.2$

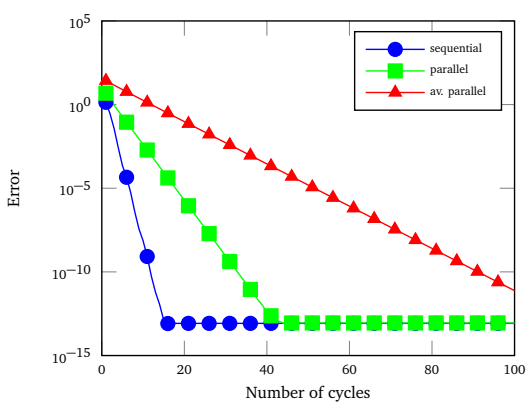

(b) $l=4$

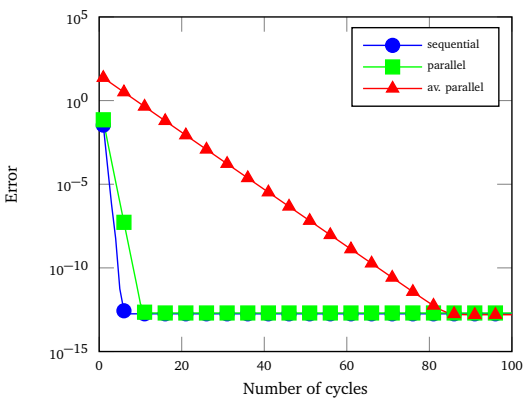

(c) $l=8$

Figure 2: Relative error of the method of reflections $\ell^{2}$-norm as a function of the number of cycles for three chosen values of the distance between the objects.

We note that the convergence is linear and that its rate increases with the value of $l$. The rate of convergence of the sequential form is also always higher than that of the averaged parallel form. This is not a surprise, as the convergence of the method of alternating projections is generally ${ }^{17}$ faster than that of its simultaneous counterpart. Also, the equal weights used in the averaging of the reflections may not constitute an optimal choice with respect to the rate of convergence. Moreover, from an effective computational perspective, the sequential form of the method may not be the most efficient one when a large number of objects is involved, since the implementation of the parallel variants can be achieved in practice using parallelisation. Nevertheless, a quantitative study of the trade-off between the parallelisation and the rates of convergence of the different forms of the method is out of the scope of the present work.

\subsection{Mixed boundary conditions: a case of divergence of the sequential form}

Since Theorem 4.1 only ensures unconditional convergence of the sequential form of the method of reflections in an orthogonal setting, cases of divergence are expected for boundary value problems falling outside of this framework, but may prove elusive. Such a configuration was obtained in two dimensions by considering a ball of radius equal to 2 centred at the origin, on which a Dirichlet boundary condition with constant datum is imposed, surrounded by a Cshaped set, on which a Neumann boundary condition with constant datum is imposed, both contained in the bounded domain previously considered. The setting of this example is shown in Figure 3.

In the numerical experiments, none of the three forms of the method converged for such a configuration.

\subsection{Influence of the distance in an unbounded domain}

The asymptotic behaviour of the rate of convergence of the method seen as a function of the distance between the objects is finally investigated. To do this, the Laplace problem dealt with in Subsection 5.1 is recast as an exterior problem set in $\mathbb{R}^{2}$, considering Neumann boundary conditions satisfying instead of Dirichlet ones, chosen such a way that the solution tends to zero at infinity. The distance $l$ between the objects being fixed, the corresponding contraction coefficient $K(l)$ is defined by

$$
K(l)=\lim _{k \rightarrow+\infty} \frac{\left\|u^{(k+1)}-u\right\|}{\left\|u^{(k)}-u\right\|},
$$

where $\|\cdot\|$ is the $\ell^{2}$-norm of a set of values of the considered function at a finite number of points on the boundaries of the objects, and estimated in practice by fitting the error as a function of the iteration. The results are presented in Figure 4. The convergence rate of the averaged parallel form appears to be asymptotically independent of the distance between the objects, but the theoretical proof of such a result is an open question.

\footnotetext{
${ }^{17}$ This fact can be commonly observed in practice (see $\left[\mathrm{CCC}^{+} 12\right]$ for instance) and is theoretically proved for $N=2$ in [RZ17].
} 

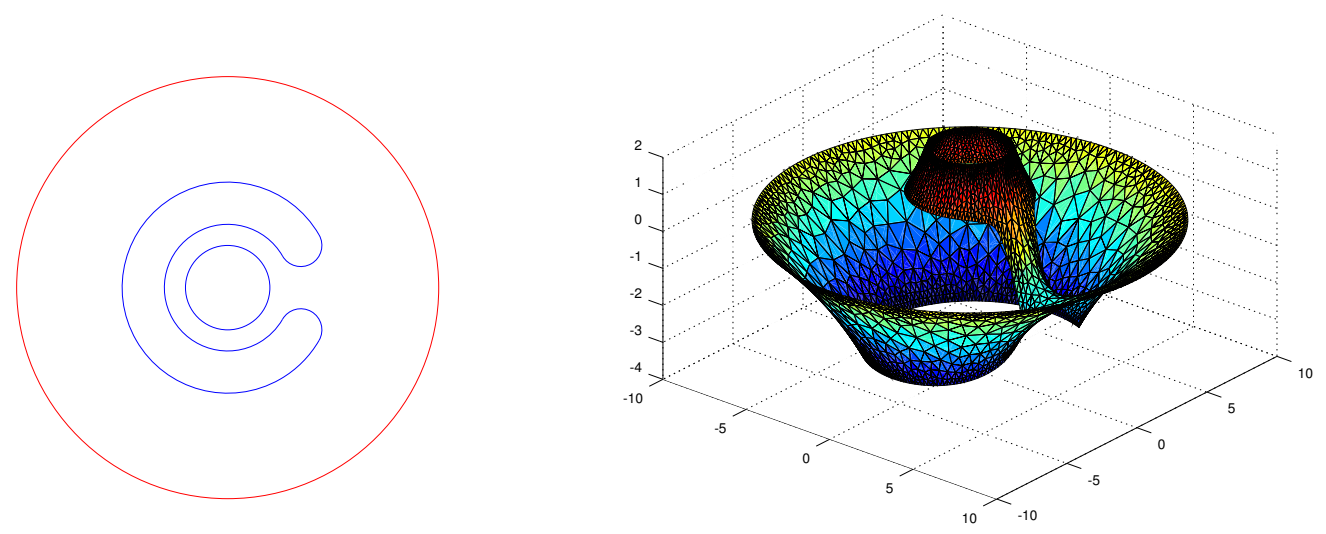

Figure 3: Example of configuration giving rise to a divergence of the method of reflections when applied to the solution of a Laplace problem with mixed (Dirichlet and Neumann) boundary conditions. Left figure: representation of the domain (with red boundary) and two objects (with blue boundaries). Right figure: numerical representation of the computed reference solution for this problem.

More generally, scalability issues, that is the analysis of the rate of convergence as a function of the number of the objects involved in the problem, is out of the scope of this paper. Note that settings for which a decomposition method achieves scalability have already been exhibited for Schwarz-type methods, see, e.g., [CG17], but remains an open question in the context of the method of reflections.

\section{Acknowledgements}

Philippe Laurent would like to thank Frédéric Boyer for introducing him to the method of reflections. Guillaume Legendre would like to thank Christophe Hazard for pointing him to the relevant paper [Bal04], and Mikhael Balabane himself for an interesting discussion on the topic. Julien Salomon would like to thank Gabriele Ciaramella, Olivier Glass and Alexandre Munnier for helpful discussions. Finally, the authors collectively thank the anonymous reviewers whose comments and suggestions helped improve the manuscript.

\section{References}

[AGG97] C. Amrouche, V. Girault, and J. Giroire. Dirichlet and Neumann exterior problems for the $n$-dimensional Laplace operator. An approach in weighted Sobolev spaces. J. Math. Pures Appl., 76(1):55-81, 1997. DoI: 10.1016/S0021-7824 (97)89945-X.

[Aro50] N. ARonsZAJN. Theory of reproducing kernels. Trans. Amer. Math. Soc., 68(3):337-404, 1950. DoI: 10. 1090/S0002-9947-1950-0051437-7.

[Aus76] A. AusLENDER. Optimisation : méthodes numériques. Masson, 1976.

[Bal04] M. BALABANE. Boundary decomposition for Helmholtz and Maxwell equations 1: disjoint sub-scatterers. Asymptotic Anal., 38(1):1-10, 2004.

[BB96] H. H. BAUSCHKE and J. M. BORWEIN. On projection algorithms for solving convex feasibility problems. SIAM Rev., 38(3):367-426, 1996. DOI: 10.1137/S0036144593251710.

[BDH09] H. H. BAuschKe, F. DeUtsch, and H. Hundal. Characterizing arbitrarily slow convergence in the method of alternating projections. Internat. Trans. Oper. Res., 16(4):413-425, 2009. DOI: $10.1111 / \mathrm{j} .1475$ 3995.2008.00682.x.

[BDH ${ }^{+}$03] H. H. BauschKe, F. DeUtsch, H. Hundal, and S.-H. PARK. Accelerating the convergence of the method of alternating projections. Trans. Amer. Math. Soc., 355(9):3433-3461, 2003. DOI: 10.1090/S0002-994703-03136-2.

[BGJ ${ }^{+}$12] F. Boyer, P. B. Gossiaux, B. Jawad, V. Lebastard, and M. Porez. Model for a sensor inspired by electric fish. IEEE Trans. Robot., 28(2):492-505, 2012. DOI: 10.1109/TR0.2011.2175764. 


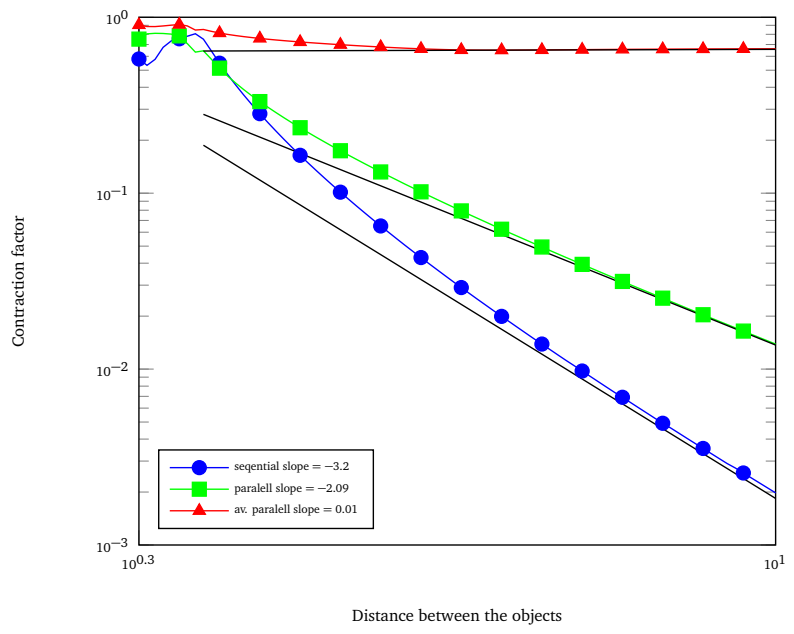

Figure 4: Plot of the value of the estimated contraction factor of the method as a function of the distance between the objects. The linear regressions are done using values associated with the five largest considered distances and are represented by black solid lines.

[BGM12] C. BADEA, S. GRIVAUX, and V. MÜLLER. The rate of convergence in the method of alternating projections. St. Petersburg Math. J., 23(3):413-434, 2012. DOI: 10.1090/S1061-0022-2012-01202-1.

[BT97] M. Balabane and V. TiRel. Décomposition de domaine pour un calcul hybride de l'équation de Helmholtz. C. R. Acad. Sci. Paris Sér. I Math., 324(3):281-286, 1997. DoI: 10.1016/S0764-4442 (99)80361-9.

$\left[\mathrm{CCC}^{+} 12\right]$ Y. CENSOR, W. CHEN, P. L. CombetTes, R. DAVIDI, and G. T. HeRMAn. On the effectiveness of projection methods for convex feasibility problems with linear inequality constraints. Comput. Optim. Appl., 51(3):10651088, 2012. DOI: $10.1007 / \mathrm{s} 10589-011-9401-7$.

[CG17] G. Ciaramella and M. J. GandER. Analysis of the parallel Schwarz method for growing chains of fixed-size subdomains: part I. SIAM J. Numer. Anal., 55(3):1330-1356, 2017. DOI: 10.1137/16M1065215.

[CGH ${ }^{+}$19] G. Ciaramella, M. J. GANDER, L. HalPern, and J. SAlOMOn. Methods of reflections: relations with Schwarz methods and classical stationary iterations, scalability and preconditioning. SMAI J. Comput. Math., 5:161193, 2019. DOI: 10.5802 /smai-jcm. 46.

[CH13] M. CASSIER and C. HAZARD. Multiple scattering of acoustic waves by small sound-soft obstacles in two dimensions: mathematical justification of the Foldy-Lax model. Wave Motion, 50(1):18-28, 2013. DOI: 10.1016/j . wavemoti.2012.06.001.

[Cim38] G. Cimmino. Calcolo approssimato per le soluzioni dei sistemi di equazioni lineari. La Ricerca Scientifica, 9:326-333, 1938.

[CJ12] J. COATLÉVEN and P. JOLY. Operator factorization for multiple-scattering problems and an application to periodic media. Commun. Comput. Phys., 11(2):303-318, 2012. DoI: 10.4208/cicp.231109.090710s.

[CK88] S. B. CHEN and H. J. KeH. Electrophoresis in a dilute dispersion of colloidal spheres. AIChE J., 34(7):10751085, 1988. DOI: 10.1002 /aic. 690340704.

[Deu85] F. DEUTSCH. Rate of convergence of the method of alternating projections. In B. BROSOWSKI and F. DEUTSCH, editors, Parametric Optimization and Approximation, volume 72 of International series of numerical mathematics, pages 96-107. Birkhäuser-Verlag, 1985.

[Deu92] F. DEUTSCH. The method of alternating orthogonal projections. In S. P. SINGH, editor, Approximation theory, spline functions and applications. Volume 356, NATO ASI Series, pages 105-121. Springer Netherlands, 1992. DOI: $10.1007 / 978-94-011-2634-2 \_5$.

[DH10] F. DEUTSCH and H. HunDAL. Slow convergence of sequences of linear operators II: arbitrarily slow convergence. J. Approx. Theory, 162(9):1717-1738, 2010. DoI: 10.1016/j . jat.2010.05.002.

[DH97] F. DEUTSCH and H. Hundal. The rate of convergence for the method of alternating projections, II. J. Math. Anal. Appl., 205(2):381-405, 1997. DOI: 10.1006/jmaa.1997.5202. 
[Dho96] J. K. G. Dhont. An introduction to dynamics of colloids, volume 2 of Studies in interface science. Elsevier, 1996.

[Dix49] J. Dixmier. Étude sur les variétés et les opérateurs de Julia, avec quelques applications. Bull. Soc. Math. France, 77:11-101, 1949.

[DL00] R. DAUTRAY and J.-L. Lions. Mathematical analysis and numerical methods for science and technology. Volume 4 Integral equations and numerical methods. Springer, 2000. DOI: 10.1007/978-3-642-61531-3.

[Fol45] L. L. FolDy. The multiple scattering of waves. I. General theory of isotropic scattering by randomly distributed scatterers. Phys. Rev., 67(3-4):107-119, 1945. DOI: 10.1103/PhysRev .67.107.

[Fri37] K. FRIEDRICHS. On certain inequalities and characteristic value problems for analytic functions and for functions of two variables. Trans. Amer. Math. Soc., 41(3):321-364, 1937. DOI: 10 .1090/S0002-99471937-1501907-0.

[Gan08] M. J. GANDER. Schwarz methods over the course of time. Electron. Trans. Numer. Anal., 31:228-255, 2008.

[GH09] M. GANESH and S. C. HAWKins. A high-order algorithm for multiple electromagnetic scattering in three dimensions. Numer. Algor., 50(4):469-510, 2009. DOI: 10.1007/s11075-008-9238-z.

[Gol34] G. M. Golusin. Auflösung einiger ebenen Grundaufaben der mathematischen Physik im Fall der Laplaceschen Gleichung und mehrfachzusammenhängender Gebiete, die durch Kreise begrenzt sind. Mat. Sb., 41(2):246276, 1934.

[Gri85] P. GrISVARD. Elliptic problems in nonsmooth domains. Pitman, 1985.

[Hal62] I. HALPERIN. The product of projection operators. Acta Sci. Math. (Szeged), 23(1-2):96-99, 1962.

[HB83] J. HAPPEL and H. BRENNER. Low Reynolds number hydrodynamics with special applications to particulate media, volume 1 of Mechanics of fluids and transport processes. Martinus Nijhoff publishers, 1983. DOI: 10.1007/978-94-009-8352-6.

[Höf18] R. HöFER. Sedimentation of inertialess particles in Stokes flows. Comm. Math. Phys., 360(1):55-101, 2018. DOI: $10.1007 / \mathrm{s} 00220-018-3131-y$.

[Hru72] E. J. HRusLov. The method of orthogonal projections and the Dirichlet problem in domains with a finegrained boundary. Math. USSR-Sb., 17(1):37-59, 1972. DOI: 10.1070/SM1972v017n01ABEH001490.

[HV18] R. M. HÖFER and J. J. L. VELÁzQUEz. The method of reflections, homogenization and screening for Poisson and Stokes equations in perforated domains. Arch. Rational Mech. Anal., 227(3):1165-1221, 2018. DOI: $10.1007 / \mathrm{s} 00205-017-1182-4$.

[IB01] K. ICHIKI and J. F. BRADY. Many-body effects and matrix inversion in low-Reynolds-number hydrodynamics. Phys. Fluids, 13(1):350-353, 2001. DOI: 10.1063/1.1331320.

[JO04] P.E. JABIN and F. OTTO. Identification of the dilute regime in particle sedimentation. Comm. Math. Phys., 250(2):415-432, 2004. DOI: $10.1007 /$ s00220-004-1126-3.

[Jon78] R. B. JonEs. Hydrodynamic interaction of two permeable spheres I: The method of reflections. Phys. A, 92(3-4):545-556, 1978. DOI: 10.1016/0378-4371(78)90150-4.

[Jor75] C. JoRdan. Essai sur la géométrie à n dimensions. Bull. Soc. Math. France, 3:103-174, 1875. DoI: 10 . $24033 /$ bsmf . 90.

[KK91] S. KIM and S. J. KARRILA. Microhydrodynamics: principles and selected applications. Butterworth-Heinemann, 1991.

[KW88] S. KAYALAR and H. L. WEINERT. Error bounds for the method of alternating projections. Math. Control Signals Systems, 1(1):43-59, 1988. DOI: 10.1007/BF02551235.

[Kyn59] G. J. KYNCH. The slow motion of two or more spheres through a viscous fluid. J. Fluid Mech., 5(2):193-208, 1959. DOI: $10.1017 / \mathrm{S} 0022112059000155$.

[Lax51] M. LAX. Multiple scattering of waves. Rev. Mod. Phys., 23(4):287-310, 1951. DoI: 10.1103/RevModPhys . 23.287.

[Lax52] M. LAx. Multiple scattering of waves. II. The effective field in dense systems. Phys. Rev., 85(4):621-629, 1952. DOI: 10.1103/PhysRev.85.621.

[Li89] T. LI. A class of non-local boundary value problems for partial differential equations and its applications in numerical analysis. J. Comput. Appl. Math., 28:49-62, 1989. DOI: 10.1016/0377-0427 (89) 90320-8. 
[LM72] J. L. LIONS and E. MAGENES. Non-homogeneous boundary value problems and applications. Volume I, volume 181 of Grundlehren der mathematischen Wissenschaften. Springer-Verlag, 1972. DOI: 10.1007/9783-642-65161-8.

[Luk89] J. H. C. LUKE. Convergence of a multiple reflection method for calculating Stokes flow in a suspension. SIAM J. Appl. Math., 49(6):1635-1651, 1989. DOI: 10.1137/0149099.

[ML11] C. MetTot and E. LAUGA. Energetics of synchronized states in three-dimensional beating flagella. Phys. Rev. E, 84(6):061905, 2011. DOI: 10.1103/PhysRevE.84.061905.

[NS20] B. NiETHAMMER and R. SCHUBERT. A local version of Einstein's formula for the effective viscosity of suspensions. SIAM J. Math. Anal., 52(3):2561-2591, 2020. DOI: 10.1137/19M1251229.

[Pie84] G. PIERRA. Decomposition through formalization in a product space. Math. Programming, 28(1):96-115, 1984. DOI: $10.1007 / \mathrm{BF} 02612715$.

[RZ17] S. REICH and R. ZALAS. The optimal error bound for the method of simultaneous projections. J. Approx. Theory, 223:96-107, 2017. DOI: 10.1016/j.jat.2017.08.005.

[Sch85] G. T. Schuster. A hybrid BIE+Born series modeling scheme: generalized Born series. J. Acoust. Soc., 77(3):865-879, 1985. DOI: 10.1121/1.392055.

[Sho77] R. E. SHOWALTER. Hilbert space methods for partial differential equations, volume 1 of Monographs and studies in mathematics. Pitman, 1977.

[Smi64] V. I. SMIRNov. A course of higher mathematics. Vol. 4, volume 61 of International series of monographs in pure and applied mathematics. Pergamon, 1964. DOI: 10.1016/C2013-0-06630-3.

[Smo11] M. Smoluchowski. Über die Wechselwirkung von Kugeln, die sich in einer zähen Flüssigkeit bewegen. Bull. Int. Acad. Sci. Cracovie, Cl. Sci. Math. Nat., Sér. A Sci. Math.:28-39, 1911.

[SSW77] K. T. Smith, D. C. Solomon, and S. L. Wagner. Practical and mathematical aspects of the problem of reconstructing objects from radiographs. Bull. Amer. Math. Soc., 83(6):1227-1270, 1977. DOI: 10.1090/ S0002-9904-1977-14406-6.

[Tra06] S. D. TRAYTAK. Convergence of a reflection method for diffusion-controlled reactions on static sinks. Phys. A Statist. Mech. Appl., 362(2):240-248, 2006. DoI: 10.1016/j . physa.2005.03.061.

[Vis49] M. I. VISHIK. The method of orthogonal and direct decomposition in the theory of elliptic differential equations (russian). Mat. Sb., 25(67):189-234, 1949.

[vNe49] J. von Neumann. On rings of operators. Reduction theory. Ann. Math. (2), 50(2):401-485, 1949. DoI: 10.2307/1969463.

[Wey40] H. WeYL. The method of orthogonal projection in potential theory. Duke Math. J., 7(1):411-444, 1940. DOI: $10.1215 / \mathrm{S} 0012-7094-40-00725-6$.

[Wil13] H. J. Wilson. Stokes flow past three spheres. J. Comput. Phys., 245:302-316, 2013. DoI: 10 . 1016/j . jcp.2013.03.020.

[WL13] H. WANG and J. LIU. On decomposition method for acoustic wave scattering by multiple obstacles. Acta Math. Sci., 33(1):1-22, 2013. DOI: 10.1016/S0252-9602(12)60191-X.

[XZ02] J. XU and L. ZiKaTANOV. The method of alternating projections and the method of subspace corrections in Hilbert space. J. Amer. Math. Soc., 15(3):573-597, 2002. DoI: 10.1090/S0894-0347-02-00398-3.

[Zar27] S. ZAREMBA. Sur un problème toujours possible comprenant, à titre de cas particulier, le problème de Dirichlet et celui de Neumann. J. Math. Pures Appl. (9), 6:127-164, 1927. DoI: 10 .1090/S0894-0347-0200398-3. 